\title{
Vegetation-environment relationships in south Nile Delta
}

\author{
Kamal H. Shaltout*, Loutfy M. Hassan** \& Emad A. Farahat** \\ *Department of Botany, Faculty of Science, Tanta University \\ **Department of Botany and Microbiology, Faculty of Science, Helwan University
}

Shaltout, K.H., Hassan, L.M. \& Farahat, E.A. 2005. Vegetation-environment relationships in south Nile Delta. Taeckholmia 25: 15-46.

\begin{abstract}
The present study aimed at identifying and analysing the plant communities of the different habitats in south Nile Delta. Two hundred and seventy one stands were selected to represent the variation in seven major types of habitat recognized in the study area (terraces, slopes, water edges, open water, gardens and nurseries, croplands and ditches, fallow and flooded lands. One hundred and forty four species belonging to 110 genera, 43 families and 23 orders were recorded. Species-rich families were Gramineae followed by Compositae, Cyperaceae, Euphorbiaceae, Leguminosa, Polygonaceae and Amaranthaceae. Thirteen vegetation groups were recognized after the application of TWINSPAN. Their ordination using DECORANA indicated moisture and human impact gradients. The moisture gradient starts with the xerophytes or drought-tolerant plants that inhabited the dry terraces and slopes of the water courses and ruderal habitats (e.g. Alhagi graecorum, Imperata cylindrica, Phragmites australis and Pluchea dioscorides groups). Then, passing through the mesophytes that inhabited the ditches, gardens and croplands (e.g. Chenopodium ficifolium, Cynodon dactylon-Paspalum dilatatum and Cyperus rotundus-Cynodon dactylon groups), and the species that inhabited the water edges (e.g. Phragmites australis, Pluchea dioscorides, Sorghum bicolor and Cyperus articulatus-Cynodon dactylon groups).The pure aquatic communities that inhabited the open water (e.g. Eichhornia crassipes-Myriophyllum spicatum, Vossia cuspidata and Vossia cuspidata-Eichhornia crassipes groups) represented the other extreme end. The correlations between the soil and water variables on one hand, and the distribution of common species in the study area on the other hand were assessed.
\end{abstract}

Keywords: Diversity, multivariate analysis, south Nile Delta, species composition.

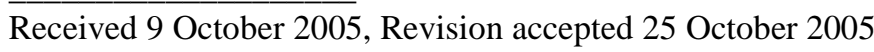




\section{Introduction}

Weeds represent biologically important components of their environments. Their persistence is remarkable in view of the efforts to eliminate them, and warrants greater attention (Radosevich \& Holt, 1984). Management, control and phytosociology of weeds have a great interest all over the world. In Egypt, many studies were carried out on the phytosociology of weeds in different Governorates (e.g. Tadros \& Atta, 1958; Springuel, 1981; El-Bakry, 1982; Hussein, 2000; Shehata \& El Fahar, 2000 and Goma'a, 2002). Nile Delta with its three sections: north, middle and south, is one of the phytogeographical regions of Egypt, which received great attention for studying their weed phytosociology (El-Shayeb, 1984 and 1989; Shaltout \& Sharaf El-Din, 1988; Shaltout \& El-Fahar, 1991; Shaltout \& El-Sheikh, 1991and 1993; Shaltout et al., 1992; Al-Sodany, 1992 and 1998; El-Demerdash et al., 1997; El-Halawany, 2000; Sheded \& Turki, 2000 and Ahmed, 2003).

The habitats recognized in the study area are: terraces, slopes, water edges and open water of the watercourses, gardens and nurseries of ElQanatir Public Park, croplands, water ditches, fallow and flooded lands. Watercourse habitats are wide spread due to the presence of the two Nile branches, effluents and the main irrigation canals. On the other hand, croplands are limited and increase northwards. The selected nurseries in the present study lie inside the gardens, so it was considered with the gardens as one habitat. Ditches, fallow lands (adjacent to the croplands and receive their water drainage) and flooded lands (inundated frequently with water of River Nile) are characterized by high moisture content and human impacts all over the year; so, they collectively considered as one habitat.

The establishment of El-Qanatir Public Park was synchronized with the construction of Delta Barrages. In the beginning, it was part of Khedive Said's castle which was constructed at El-Qanatir to be used as fortress against any navy may attack the state through the Nile (Zaki, 1947). Nowadays, The Park represents the northern lung of the greater Cairo and its boundaries in addition to the internal and external tourism. The relatively large green area of the gardens ( $35 \mathrm{ha}$ ) gives it its potentialities as public park.

The weeds of the southernmost part of the south Nile Delta and its surroundings seem to be poorly studied. The objectives of the present study were to determine the structure of the weed vegetation in terms of spatial and temporal variations in the floristic composition and abundance of 
species in South Nile Delta (El-Qanatir region), to analyze the spatial and temporal variations in the environmental factors (soil, water, climate and human impacts) that affect the species and community distribution and to assess the degree of correlation between the environmental and vegetation variables.

\section{Study area}

The study area is located in the most southern sections of the South Nile Delta (between latitude $30^{\circ} 10^{\prime} 27^{\prime \prime} \mathrm{N}$ to $30^{\circ} 14^{\prime} 36^{\prime \prime} \mathrm{N}$ and longitude $31^{\circ} 3{ }^{\prime}$ 9" E to $31^{\circ} 9^{\prime} \mathrm{E}$ ). It extends from about $20 \mathrm{~km}$ north of Cairo northwards to south of Menoufia Governorate and extending from Tawfiky effluent in the east to Behera and Nasery effluents in the west (Fig. 1). In addition to the two branches of the River Nile, the study area is dissected by four effluents. They feed the three divisions of the Delta: the western Behira and Nasery effluents, the central Menoufy effluent and the eastern Tawfiky effluent. On the two branches of the Nile and their effluents, a number of barrages were built for controlling the water discharge budgets. From these watercourses, many irrigation canals are branched.

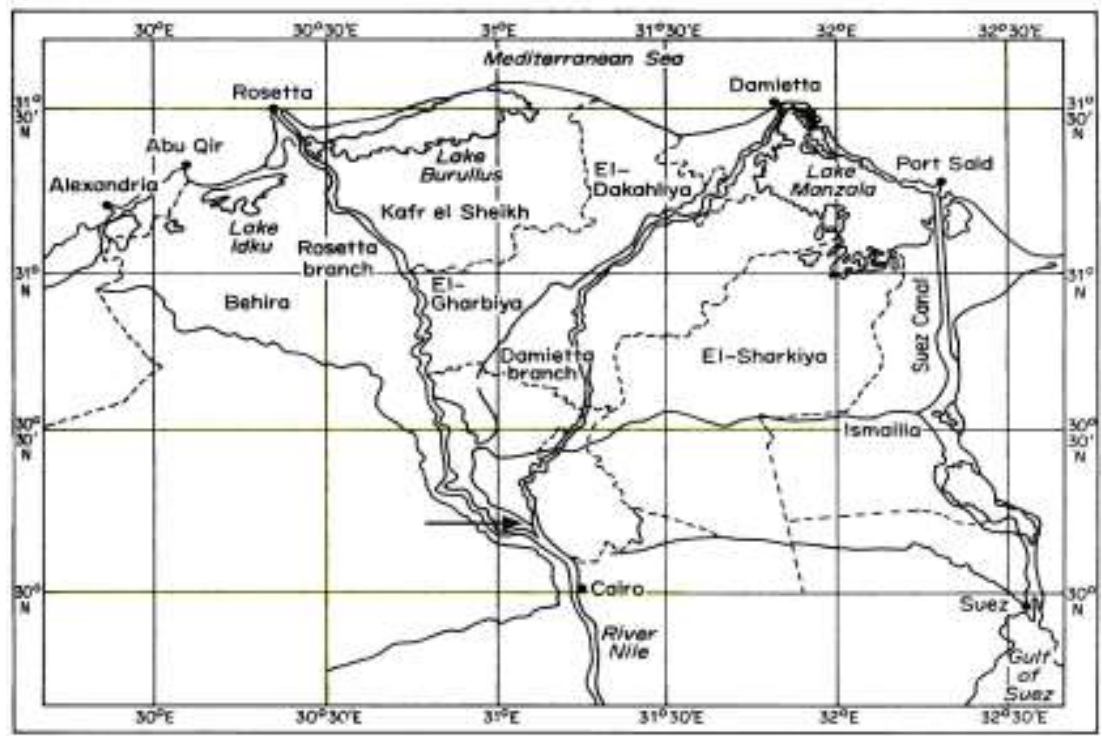

Fig. 1. The main features of the Nile Delta (arrow refers to the study area, c.f. Zahran \& Willis, 1992). 
Table 1. Long-term annual averages (1960-1975) of the meteorological data of two meteorological stations in the study area (Anonymous, 1980).

\begin{tabular}{|l|cc|cc|}
\hline \multirow{2}{*}{ Meteorological variables } & \multicolumn{2}{|c|}{ BANHA } & \multicolumn{2}{c|}{ CAIRO } \\
\cline { 2 - 5 } & $31^{\circ} 11^{\prime} \mathrm{E}, 30^{\circ} 28^{`} \mathrm{~N}$ & $31^{\circ} 15^{`} \mathrm{E}, 30^{\circ}$ & $03^{`} \mathrm{~N}$ \\
\cline { 2 - 5 } & Range & Mean & Range & Mean \\
\hline Max. air temperature $\left({ }^{\circ} \mathrm{C}\right)$ & $19.3-34.4$ & 27.8 & $19-34.9$ & 28 \\
Min. air temperature $\left({ }^{\circ} \mathrm{C}\right)$ & $7.6-20.8$ & 14.4 & $8.8-21.8$ & 15.6 \\
Mean air temperature $\left({ }^{\circ} \mathrm{C}\right)$ & $13.4-27.4$ & 21.1 & $13.9-28.3$ & 21.4 \\
Relative humidity $(\%)$ & $48-75$ & 76 & $42-61$ & 53 \\
Evaporation $\left(\mathrm{mm} \mathrm{day}^{-1}\right)$ & $2.9-8.4$ & 5.2 & $7.4-17$ & 11.8 \\
Rainfall (mm year $\left.{ }^{-1}\right)$ & - & 1.9 & - & 1.9 \\
\hline
\end{tabular}

\section{Materials and methods}

\section{a) Vegetation}

Two hundred and seventy one stands were selected to represent the apparent variation in the different habitats in the study area which include: terraces, slopes, water edges and open water of the watercourses; gardens and nurseries; croplands; and ditches, fallow and flooded lands. The numbers of stands sampled in each habitat varies between 22 in the ditches, fallow and flooded lands and 62 along the edges of the watercourses (depending on the micro variation in physiography and vegetation physiognomy). The area of each stand was about $20 \times 20 \mathrm{~m}$ or according to the extension of plant cover and / or the type of the selected habitat.

The following parameters were determined in each stand: seasonal listing of the species indicating the first and second dominant species, visual estimating of the total cover and the cover of each species (\%), and the physical changes occurring (grazing, firing, removing of the plants and human impacts). Nomenclature was according to Täckholm (1974), Boulos (1995, 1999, 2000 and 2002). 


\section{b) Soil and water}

Three soil samples were collected from profiles $(0-50 \mathrm{~cm})$ of each sampled stand. Soil texture was determined by the Bouyoucous hydrometer method. Calcium carbonate was estimated using Bernard's calcimeter. Soil water extracts of 1:5 were prepared for the determination of soil salinity (EC) and soil reaction $(\mathrm{pH})$ using electric conductivity $\left(\mathrm{mS} \mathrm{cm} \mathrm{cm}^{-1}\right)$ and $\mathrm{pH}$ meters. Chlorides were determined by direct titration against silver nitrate solution using $5 \%$ potassium chromate as an indicator. Soluble bicarbonates were estimated by titration against $0.01 \mathrm{~N} \mathrm{HCl}$, and sulphates were determined turbidimetrically as barium sulphate at $500 \mathrm{~nm}$. Nitrates were determined using sodium salicylate, $\mathrm{H}_{2} \mathrm{SO}_{4}$ and $\mathrm{NaOH}$ as analytical reagents. The sulphanilamide diazotization was used for determination of nitrite in soil extract. Phosphorus was determined in the soil water extract by the direct colorimetric Molybdenum blue method. $\mathrm{Ca}$ and $\mathrm{Mg}$ were determined by titration against $0.01 \mathrm{~N}$-versenate solution using meroxide and erichrome black $\mathrm{T}$ as indicators. Sodium and potassium were determined using flame photometer (Allen et al., 1974).

Water samples were collected from the stands of the open water and the edges of the watercourses. Determination of $\mathrm{pH}, \mathrm{EC}$ and dissolved oxygen were carried out directly after collection. Concentration of dissolved oxygen in water samples (as ppm) was measured using a glass electrode dissolved oxygen meter. Soluble carbonates in water, $\mathrm{NO}_{3}, \mathrm{NO}_{2}, \mathrm{P}, \mathrm{SO}_{4}, \mathrm{~K}, \mathrm{Na}, \mathrm{Ca}$, and $\mathrm{Mg}$ were estimated using the same methods of soil analysis (Allen et al., 1974).

\section{c) Data analysis}

Two-way indicator species analysis (TWINSPAN) and detrended correspondence analysis (DECORANA) were applied to the matrix of cover estimates of 144 species in 271 stands (Hill, 1979 a,b). The agglomerative clustering techniques were applied to ordinate and classify the zonal vegetation of the habitats based on Sørensen similarity coefficient. The Sørensen similarity matrix was plotted using the Non-Metric Multidimensional scaling (N-MDS) technique (Kruscal, 1964). Species richness for vegetation groups was calculated as the average number of species per stand. Species turnover was calculated as a ratio between the total number of species recorded in a certain vegetation group and its alpha diversity (Whittaker, 1972). Relative evenness of the importance value of species was expressed by Shannon-Wiener index: $\hat{\mathrm{H}}=-\sum_{\mathrm{i}=1}^{\mathrm{s}} \mathrm{Pi}(\log \mathrm{Pi})$. The 
relative concentration of dominance was expressed by Simpson's index: $\mathrm{D}=1 / \mathrm{C}, \mathrm{C}=-\sum_{\mathrm{i}=1}^{\mathrm{s}}(\mathrm{Pi})^{2}$, where $\mathrm{S}$ is the total number of species and $\mathrm{Pi}$ is the relative importance value (relative cover) of $i^{\text {th }}$ species (Pielou, 1975; Magurran, 1988). Principal Components Analysis (PCA) is a technique used to summarise the relationship between the species of the plant communities and the soil variables in the form of simple figure (Kent \& Coker, 1992) in which numbers and squares represent the vegetation groups and lines represent the soil variables. Simple linear correlation coefficient (r) was calculated for assessing the relationship between the estimated soil and water variables on one hand, and the community variables of the vegetation groups on the other hand. The variation in the soil and water variables in relation to the vegetation groups were assessed using one-way analysis of variance (ANOVA). These techniques were according to SPSS Software (SPSS, 1999).

\section{Results}

One hundred and forty four species belonging to 110 genera, 43 families and 23 orders were recorded in the study area. The most characteristic families are Gramineae (20.9 \%) followed by Compositae (13.9 \%), Cyperaceae and Euphorbiaceae (5.6 \% for each), Leguminosae and Polygonaceae (4.9\% for each) and Amaranthaceae (4.2\%) (see Tables 2 and 3). The species recorded only along the terraces of watercourses were Enarthrocarpus lyratus, Tamarix nilotica and Trigonella hamosa, while Ceruana pratensis recorded only along the slopes (Table 4). Lantana camara and Persicaria lanigera recorded only along the water edge, and Lemna gibba, Potamogeton crispus, $P$. nodosus and $P$. pectinatus in the open water. Commicarpus helenae, Eragrostis cilianensis, Lotus glaber, Mentha piperitta, Allium roseum, Paspalum dilatatum and Polygonum equisetiforme were recorded only in the gardens and nurseries. Some other species were recorded only in the ditches, flooded and fallow lands (e.g. Abutilon theophrasti, Cyperus difformis and Fimbristylis bes-umbellata), and croplands (e.g. Amaranthus spinosus, Cenchrus echinatus, Coronopus squamatus, Eragrostis pilosa, and Malvastrum coromandelianum)

The application of the agglomerative clustering and non-metric multidimensional scaling (ordination) technique on the plant communities of the seven habitats (Fig. 2) indicated a distinction of five clusters. The first cluster comprised the terraces and slopes communities, while the second and third clusters represented the communities of the water edges, gardens and 
nurseries, respectively. The communities of croplands, ditches, fallow and flooded lands were represented by the fourth cluster and that of the open water by the fifth one. The polar ordination revealed these relationships in an impressive manner (Fig. 2b); in which the terrestrial habitats (gardens, croplands and ditches) segregated away from the watercourses habitats. By turn, the watercourses were segregated into three clusters; the open water at the upper left side of the diagram and the terraces and slopes at the lower left side; while the water edges were in transitional position among them.

The life form spectra of the recorded species (Table 5) indicated that therophytes have the highest contribution to the total flora of the study area $(58.3 \%)$, followed by the geophytes-helophytes $(20.8 \%)$. The variation in relation to the types of habitat indicated that the therophytes have the highest contribution in the croplands $(80.8 \%)$, chamaephytes and hemicryptophytes in the terraces (each of $8.4 \%$ ), geophytes-helophytes $(32.1 \%)$ and hydrophytes $(28.61 \%)$ in the open water.

Seasonal variations in the number of the recorded species per habitat (Table 6) indicated that, generally, the highest number of species (123 species) was recorded in spring season. The open water had the lowest numbers of the recorded species all the year around, while ditches, fallow and flooded lands had the highest numbers of species in spring and summer (71 and 60 species, respectively). Moreover, the water edges had the highest numbers of species in spring (57 species) and winter (58 species). The highest numbers of species per year were recorded in the ditches, fallow and flooded lands (97 species), followed by the water edges ( 84 species) and croplands (78 species). The distribution of the recorded species in the different seasons showed that $52.1 \%$ of the recorded species were winter active species, $20.8 \%$ were summer active species, $85.4 \%$ were spring active species and $27.1 \%$ are all-year active species.

The application of TWINSPAN classification technique on the cover estimates of 144 species in 271 stands led to the recognition of 38 vegetation groups at level 6 of the classification. The application of DCA on the same set of data indicated a reasonable segregation among these groups along the ordination axes 1 and 2 (Fig. 3). These groups could be categorized at level 3 and 4 of the classification into 13 major groups (Fig. 3 , Table 7). These groups were named after their dominant species as follows: (I) Alhagi graecorum in the habitat of terraces, slopes and fallow lands, (II) Persicaria senegalensis-Alhagi graecorum in the flooded lands, (III) Pluchea dioscorides in the water edges, (IV) Imperata cylindrica in the 
slopes, water edges and fallow lands, (V) Eichhornia crassipesMyriophyllum spicatum in the open water, (VI) Vossia cuspidataEichhornia crassipes in the open water and water edges, (VII) Vossia cuspidata in the open water, (VIII) Phragmites australis in many habitats, (IX) Sorghum bicolor in the water edges (X) Chenopodium ficifolium in the croplands, (XI) Cyperus rotundus-Cynodon dactylon in the gardens, nurseries, ditches and fallow lands, (XII) Cynodon dactylon-Paspalum dilatatum in the gardens and nurseries, and (XIII) Cyperus articulatusCynodon dactylon in the flooded lands, water edges and terraces of watercourses (Table 7).

Species richness of the vegetation groups varied between 87 species in Cyperus rotundus-Cynodon dactylon group (XI) and 3 species in Sorghum bicolor group (IX) (Table 8). VG XI had the highest values of species richness (11.6), relative concentration of dominance (37.4) and relative evenness (4.0). The highest value of species turnover (13.1) was recorded in the Phragmites australis group (VIII). The Sorghum bicolor group (IX) had the lowest values of relative concentration of dominance (3.0) and relative evenness (1.1), while VG II and V had the lowest values of species richness (2.2) and species turnover (1.0), respectively. The relationship between species richness and species dominance was expressed in the form of dominance-diversity curves (Fig. 4). It is clear that most of the curves were steep and the dominance was represented by one or a few species.

Most of the soil variables of the vegetation groups differed significantly according to the one-way ANOVA (Table 9). Soil of Alhagi graecorum group (I) had the highest values of EC $\left(5.2 \mathrm{mS} \mathrm{cm}^{-1}\right)$, chloride $\left(7 \mu \mathrm{g} \mathrm{g}^{-1}\right)$ and nitrates $\left(6 \mu \mathrm{g} \mathrm{g}^{-1}\right)$. Soil of Persicaria senegalensis-Alhagi graecorum group (II) had the highest values of sand (91.9\%), phosphorus $\left(387 \mu \mathrm{g} \mathrm{g}^{-1}\right)$ and $\mathrm{pH}$ (7.9), but the minimum of clay (4.1\%). Soil of Vossia cuspidataEichhornia crassipes group (VI) had the highest values of clay (13.3\%), $\mathrm{Na}\left(37 \mu \mathrm{g} \mathrm{g}^{-1}\right), \mathrm{Ca}\left(3 \mu \mathrm{g} \mathrm{g}{ }^{-1}\right)$ and $\mathrm{CaCO}_{3}(6.8 \%)$. In addition, soil of Phragmites australis group (VIII) had the highest values of sulphate, while soil of Chenopodium ficifolium group (X) had the lowest of chloride, nitrate, phosphorus and sodium. Soil of Cyperus rotundus-Cynodon dactylon group (VG XI) had the minimum values of silt and nitrites, while soil of Cyperus articulatus- Cynodon dactylon group had the minimum of $\mathrm{EC}$ and $\mathrm{CaCO}_{3}$. 
Table 2. Presence percentages $(\mathrm{P} \%)$ of the annual species in the study area. The species are arranged in descending order according to their presence percentage in all sampled stands. The habitats are: $\mathrm{TE}=$ terraces, $\mathrm{SL}=$ slopes, $\mathrm{WE}=$ water edges, $\mathrm{OW}=$ open water, $\mathrm{GR}=$ gardens and nurseries, $\mathrm{DI}=$ ditches, fallow and flooded lands and AG = croplands. The vegetation groups are: (I) Alhagi graecorum, (II) Persicaria senegalensis-Alhagi graecorum, (III) Pluchea dioscorides, (IV) Imperata cylindrica, (V) Eichhornia crassipes-Myriophyllum spicatum, (VI) Vossia cuspidata-Eichhornia crassipes, (VII) Vossia cuspidata, (VIII) Phragmites australis, (IX) Sorghum bicolar, (X) Chenopodium ficifolium, (XI) Cyperus rotundus-Cynodon dactylon, (XII) Cynodon dactylon-Paspalum dilatatum and (XIII) Cyperus articulatus-Cynodon dactylon.

\begin{tabular}{|c|c|c|c|c|}
\hline Name of species & Family & Habitat & $\begin{array}{l}\text { Vegetative } \\
\text { group }\end{array}$ & $\mathbf{P} \%$ \\
\hline Rumex dentatus $\mathrm{L}$. & Polygonaceae & TE,SL,WE,OW,DI,AG & IV, VII, VIII, XI, XII & 16.2 \\
\hline Amaranthus hybridus $\mathrm{L}$. & Amaranthaceae & TE,SL,WE,GR,DI,AG & II, VII, VIII, XI & 14.8 \\
\hline Pоа аппиа $\mathrm{L}$. & Gramineae & WE,GR,DI,AG & XI, XII & 11.8 \\
\hline Coronopus niloticus (Delile) Spreng. & Cruciferae & TE,SL,WE,GA,DI,AG & VII, VIII, XI, XII & 10.3 \\
\hline Echinochloa colona (L.) Link & Gramineae & TE,SL,WE,GR,DI,AG & VII, XI, XII, XIII & 10.3 \\
\hline Portulaca oleracea $\mathrm{L}$. & Portulacaceae & TE,SL,WE,GR,DI,AG & VII, VIII, XI & 9.6 \\
\hline Euphorbia peplus L. & Euphorbiaceae & WE, GR,DI,AG & XI, XII & 9.2 \\
\hline Chenopodium murale $\mathrm{L}$. & Chenopodiaceae & TE,SL,WE,GR,DI,AG & VIII, XI, XII & 8.9 \\
\hline Eclipta prostrata (L.) L. & Compositae & TE,SL,WE,DI & $\begin{array}{l}\text { III, IV, VI, VII, VIII, } \\
\text { XII }\end{array}$ & 8.9 \\
\hline Chenopodium ficifolium Sm. & Chenopodiaceae & TE,SL,WE,GR,DI,AG & VII, VIII, X, XI, XII & 8.5 \\
\hline Rorippa palustris (L.) Besser & Cruciferae & TE,SL,WE,OW,DI,AG & $\begin{array}{l}\text { IV, VII, VIII, X, XI, } \\
\text { XII }\end{array}$ & 8.1 \\
\hline $\begin{array}{l}\text { Pseudognaphalium luteo-album (L.) } \\
\text { Hilliard \& B.L. Burtt }\end{array}$ & Compositae & $\begin{array}{l}\text { TE,SL,WE,OW,GR,DI, } \\
\text { AG }\end{array}$ & $\begin{array}{l}\text { All except I, II, VI, } \\
\text { IX, }\end{array}$ & 7.7 \\
\hline Bidens pilosa $\mathrm{L}$. & Compositae & TE,WE,GR,DI,AG & VIII, XI, XII & 7.0 \\
\hline Malva parviflora $\mathrm{L}$. & Malvaceae & TE,SL,WE,GR,DI,AG & VII, VIII, XI, XII & 7.0 \\
\hline Sonchus oleraceus $\mathrm{L}$. & Compositae & TE,SL,WE,GR,DI,AG & I, III, VII, XI, XII & 7.0 \\
\hline Anagallis arvensis $\mathrm{L}$. & Primulaceae & GR,DI,AG & XI & 6.3 \\
\hline Bromus catharticus Vahl & Gramineae & SL,WE,GR,DI,AG & XI, XII & 5.9 \\
\hline Glinus lotoides $\mathrm{L}$. & Molluginaceae & TE,SL,WE,OW,DI & III, VII & 5.9 \\
\hline Polypogon monspeliensis (L.) Desf. & Gramineae & $\begin{array}{l}\text { TE,SL,WE,OW,GR,DI, } \\
\text { AG }\end{array}$ & $\begin{array}{l}\text { IV, VII, VIII, XI, } \\
\text { XIII }\end{array}$ & 5.9 \\
\hline Conyza bonariensis (L.) Cronquist & Compositae & TE,SL,WE,GR,DI,AG & III, VIII, XI, XII & 4.8 \\
\hline Lamium amplexicaule $\mathrm{L}$. & Labiatae & TE,GR,DI,AG & XI & 4.8 \\
\hline Trianthema portulacastrum $\mathrm{L}$. & Aizoaceae & TE,SL,GR,DI,AG & VIII, XI, XII & 4.8 \\
\hline Capsella bursa-pastoris (L.) Medik. & Cruciferae & WE,GR,DI,AG & XI, XII & 4.4 \\
\hline $\begin{array}{l}\text { Cichorium endiva subsp. divaricatum } \\
\text { (Schousb.) P.D. Sell }\end{array}$ & Compositae & TE,SL,GR,DI,AG & VIII, X, XI, XII & 4.4 \\
\hline $\begin{array}{l}\text { Fimbristylis bisumbellata (Forssk.) } \\
\text { Bubani }\end{array}$ & Cyperaceae & DI & $\begin{array}{l}\text { IV, VIII, XI, XII, } \\
\text { XIII }\end{array}$ & 4.4 \\
\hline Digitaria sanguinalis (L.) Scop. & Gramineae & WE,GR,DI,AG & XI, XII & 3.7 \\
\hline Setaria $x$ verticilliformis Dumort. & Gramineae & SL,WE,GR,D,AG & VII, VIII, XI, XII & 3.7 \\
\hline
\end{tabular}


K.H. Shaltout et al.

\begin{tabular}{|c|c|c|c|c|}
\hline Urtica urens L. & Urticaceae & WE,GR,DI,AG & XI & 3.7 \\
\hline Trifolium resupinatum $\mathrm{L}$. & Leguminosae & TE,GR,DI,AG & XI, XII & 3.3 \\
\hline Medicago polymorpha $\mathrm{L}$. & Leguminosae & TE,SL,WE,GR,AG & X, XI, XII & 3.0 \\
\hline Sisymbrium irio L. & Cruciferae & TE,WE,GR,AG & XI, XII & 3.0 \\
\hline Emex spinosa $\mathrm{L}$. & Polygonaceae & GR,DI,AG & XI, XII & 2.6 \\
\hline Setaria viridis (L.) P. Beauv. & Gramineae & TE,WE,GR,DI,AG & XI, XII & 2.6 \\
\hline Sorghum bicolor (L.) Moench. & Gramineae & TE,WE,GR,DI,AG & IX, XI & 2.6 \\
\hline Amaranthus graecizans L. & Amaranthaceae & SL,WE,DI,AG & VII, VIII, XI & 2.2 \\
\hline Eleusine indica (L.) Gaertn. & Gramineae & TE,SL,GR,DI,AG & XI, XII & 2.2 \\
\hline Euphorbia heterophylla L. & Euphorbiaceae & GR,DI,AG & XI & 2.2 \\
\hline Leptochloa panicea (Retz.) Ohwi & Gramineae & DI,AG & $\mathrm{X}, \mathrm{XI}$ & 2.2 \\
\hline $\begin{array}{l}\text { Apium leptophyllum (Pers.) F. Muell. } \\
\text { ex Benth }\end{array}$ & Umbelliferae & GR,DI,AG & $\mathrm{XI}$ & 1.8 \\
\hline Chenopodium ambrosioides L. & Chenopodiaceae & TE,SL,WE,DI,AG & VII, VIII & 1.8 \\
\hline $\begin{array}{l}\text { Homognaphalium pulvinatum (Delile) } \\
\text { Fayed \& Zareh }\end{array}$ & Compositae & TE,SL,WE,DI & VII, X, XI & 1.8 \\
\hline Stellaria pallida (Dumort.) Pire & Caryophyllaceae & $\mathrm{GR}, \mathrm{AG}$ & $\mathrm{XI}$ & 1.8 \\
\hline Amaranthus viridis L. & Amaranthaceae & GR,DI,AG & XI & 1.5 \\
\hline $\begin{array}{l}\text { Dichanthium annulatum (Forssk.) } \\
\text { Stapf }\end{array}$ & Gramineae & TE,SL,WE,GR,DI & IV, IX, XII & 1.5 \\
\hline $\begin{array}{l}\text { Ethulia conyzoides subsp. conyzoides } \\
\text { L. }\end{array}$ & Compositae & SL,WE & VIII & 1.5 \\
\hline Euphorbia prostrata Aiton & Euphorbiaceae & GR,DI,AG & XI, XII & 1.5 \\
\hline Lolium perenne L. & Gramineae & GR,DI,AG & XI, XII & 1.5 \\
\hline Phalaris minor Retz. & Gramineae & TE,SL,WE,GR,DI,AG & VII, VIII & 1.5 \\
\hline Tribulus terrestris L. & Zygophyllaceae & $\mathrm{GR}, \mathrm{AG}$ & $\mathrm{XI}$ & 1.5 \\
\hline Vicia sativa $\mathrm{L}$ & Leguminosae & TE,GR,AG & VIII, XI, XII & 1.5 \\
\hline Ammi visnaga (L.) Lam. & Umbelliferae & DI,AG & $\mathrm{XI}$ & 1.1 \\
\hline Avena fatua $\mathrm{L}$. & Gramineae & TE,DI,AG & $\mathrm{XI}$ & 1.1 \\
\hline Senecia aegyptius var. aegyptius L. & Compositae & TE,SL,WE,DI & VII & 1.1 \\
\hline Xanthium spinosum L. & Compositae & TE,SL & VIII, XII & 1.1 \\
\hline Xanthium strumarium L. & Compositae & TE,SL,AG & XI & 1.1 \\
\hline Amaranthus spinosus L. & Amaranthaceae & DI,AG & XI & 0.7 \\
\hline Cyperus difformis $\mathrm{L}$. & Cyperaceae & WE,DI & XIII & 0.7 \\
\hline Dactyloctenium aegyptium (L.) Willd. & Gramineae & WE,GR,DI,AG & XI, XIII & 0.7 \\
\hline Eragrostis pilosa (L.) P.Beauv. & Gramineae & $\mathrm{AG}$ & VIII & 0.7 \\
\hline Euphorbia hirta L. & Euphorbiaceae & $\mathrm{GR}, \mathrm{AG}$ & XI & 0.7 \\
\hline Matricaria recutita var. recutita $L$. & Compositae & GR,DI,AG & XI & 0.7 \\
\hline Melilotus indicus (L.) All. & Leguminosae & SL,WE,GR,DI,AG & XI & 0.7 \\
\hline Senecio vulgaris $\mathrm{L}$. & Compositae & SL,WE & VII & 0.7 \\
\hline Abutilon theophrasti Medik. & Malvaceae & DI & XI & 0.4 \\
\hline Amaranthus lividus L. & Amaranthaceae & DI,AG & XI & 0.4 \\
\hline Ammi majus L. & Umbelliferae & GR,DI,AG & $\mathrm{XI}$ & 0.4 \\
\hline Bergia capensis $\mathrm{L}$. & Elatinaceae & DI & XII & 0.4 \\
\hline
\end{tabular}




\section{Vegetation-environment relationships in south Nile Delta}

\begin{tabular}{|c|c|c|c|c|}
\hline Cenchrus echinatus L. & Gramineae & $\mathrm{AG}$ & $\mathrm{XI}$ & 0.4 \\
\hline Ceruana pratensis Forssk. & Compositae & SL & VIII & 0.4 \\
\hline Chrozophora plicata (Vahl) Spreng. & Euphorbiaceae & TE,SL & VIII & 0.4 \\
\hline $\begin{array}{l}\text { Eragrostis cilianensis (All.) F.T. } \\
\text { Hubb. }\end{array}$ & Gramineae & GR & XI & 0.4 \\
\hline Euphorbia helioscopia L. & Euphorbiaceae & SL,WE,DI,AG & $\mathrm{XI}$ & 0.4 \\
\hline Euphorbia indica Lam. & Euphorbiaceae & $\mathrm{AG}$ & XI & 0.4 \\
\hline Gynandropsis gynandra (L.) Briq. & Cleomaceae & DI,AG & XI & 0.4 \\
\hline Lactuca serriola $\mathrm{L}$. & Compositae & SL,WE,GR,DI & VIII & 0.4 \\
\hline $\begin{array}{l}\text { Malvastrum coromandelianum (L.) } \\
\text { Garcke }\end{array}$ & Malvaceae & AG & $\mathrm{XI}$ & 0.4 \\
\hline Ranunculus sceleratus L. & Ranunculaceae & SL,WE,OW,DI & VII & 0.4 \\
\hline $\begin{array}{l}\text { Senecio glaucus subsp. coronopifolius } \\
\text { L. (Maire) C. Alexander }\end{array}$ & Compositae & WE,GR,DI,AG & XI & 0.4 \\
\hline Setaria italica (L.) P. Beauv. & Gramineae & AG & $\mathrm{XI}$ & 0.4 \\
\hline Coronopus squamatus (Forssk.) Asch. & Cruciferae & AG & XII & 0.4 \\
\hline Enarthrocarpus lyratus (Forssk.) DC. & Cruciferae & $\mathrm{TE}$ & XIII & 0.4 \\
\hline Juncus bufonius L. var. bufonius & Juncaceae & DI & XIII & 0.4 \\
\hline Potentilla supina $\mathrm{L}$. & Rosaceae & TE,WE,DI,AG & VII & 0.4 \\
\hline Trigonella hamosa $\mathrm{L}$. & Leguminosae & $\mathrm{TE}$ & I & 0.4 \\
\hline
\end{tabular}

The water of Phragmites australis group (VIII) had the highest of EC, bicarbonate, chloride, phosphorus, sodium, calcium and magnesium (Table 10). Water of Sorghum bicolor group (IX) had the highest value of sulphate (28.3 $\mathrm{mg} \mathrm{l}^{-1}$ ), while the water of Imperata cylindrica-Pluchea dioscorides group (IV) had the minimum of sulphate $\left(14.1 \mathrm{mg} \mathrm{l}^{-1}\right)$.

The correlation between the vegetation and soil variables was demonstrated by Principal Components Analysis (PCA) ordination biplot (Fig. 5). It was clear that the vegetation group $\mathrm{V}$ occupied high position along the gradient of calcium carbonate and electric conductivity, while VG III and X occupied high and intermediate positions along the gradient of nitrate, respectively. Moreover, it was evident that VG VIII and IX occupied high positions along the gradient of clay, while VG VII and XI occurred at high positions along the gradient of sulphates and $\mathrm{pH}$, respectively.

Regarding the pairs of community variables, the total number of species was positively correlated with all the other community variables (Table 11). Also, relative evenness was positively correlated with species richness and species turnover $(r=0.74$ and 0.79 respectively), and negatively with relative concentration of dominance $(-0.62)$. Regarding the community versus soil variables, only $\mathrm{CaCO}_{3}$ was negatively correlated with species richness $(r=-0.65)$. 
Table 3. Presence percentage $(\mathrm{P} \%)$ of the perennial species in south Nile Delta. The species are arranged in descending order according to their presence percentage in all sampled stands. The habitats are: TE = terraces, $\mathrm{SL}=$ slopes, $\mathrm{WE}=$ water edges, $\mathrm{OW}=$ open water, GR = gardens and nurseries, DI = ditches, fallow and flooded lands and $\mathrm{AG}=$ croplands. The vegetation groups are: (I) Alhagi graecorum, (II) Persicaria senegalensis-Alhagi graecorum, (III) Pluchea dioscorides, (IV) Imperata cylindrica, (V) Eichhornia crassipes-Myriophyllum spicatum, (VI) Vossia cuspidata-Eichhornia crassipes, (VII) Vossia cuspidata, (VIII) Phragmites australis, (IX) Sorghum bicolar, (X) Chenopodium ficifolium, (XI) Cyperus rotundus-Cynodon dactylon, (XII) Cynodon dactylon-Paspalum dilatatum and (XIII) Cyperus articulatus-Cynodon dactylon.

\begin{tabular}{|c|c|c|c|c|}
\hline Name of species & Family & Habitat & Vegetative group & $\mathbf{P}$ \\
\hline \multicolumn{5}{|l|}{ Phanerophytes } \\
\hline Pluchea dioscorides (L.) DC. & Compositae & TE,SL,WE,GR,DI,AG & $\begin{array}{l}\text { All except I, V, IX, X, } \\
\text { XI }\end{array}$ & 15.9 \\
\hline Ricinus communis $\mathrm{L}$. & Euphorbiaceae & TE,SL,WE,OW & IV, VII, VIII & 5.9 \\
\hline Cynanchum acutum subsp. acutum L. & Asclepiadaceae & TE,SL,WE,OW,GR,DI & VIII, XII & 4.1 \\
\hline Lantana camara $\mathrm{L}$ & Verbenaceae & WE & III & 0.4 \\
\hline Tamarix nilotica (Ehrenb.) Bunge & Tamaricaceae & $\mathrm{TE}$ & I & 0.4 \\
\hline \multicolumn{5}{|l|}{ Chamaephytes } \\
\hline Solanum nigrum $\mathrm{L}$. & Solanaceae & TE,SL,WE,GR,DI,AG & IV, VII, VIII, XI & 3.7 \\
\hline $\begin{array}{l}\text { Symphyotrichum squamatum (Spreng.) } \\
\text { Hieron. }\end{array}$ & Gramineae & TE,SL,WE,DI & VII, VIII, XII & 2.2 \\
\hline Withania somnifera (L.) Dunal & Solanaceae & TE,SL,WE,GR,DI,AG & I, III, IV, VII, XI & 2.2 \\
\hline Centaurea calcitrapa $\mathrm{L}$. & Compositae & SL,WE & VII, VIII & 0.7 \\
\hline Ipomoea carnea Jacq. & Convolvulaceae & TE,GR,DI & II, VII & 0.7 \\
\hline Oxystelma esculentum (L.f.) R.Br. & Asclepiadaceae & TE,SL,WE & VIII & 0.4 \\
\hline Polygonum equisetiforme $\mathrm{Sm}$. & Polygonaceae & GR & XI & 0.4 \\
\hline \multicolumn{5}{|l|}{ Hemicryptophytes } \\
\hline Convolvulus arvensis $\mathrm{L}$. & Convolvulaceae & TE,SL,WE,GR,DI,AG & I, IV, VIII, XI, XII & 16.2 \\
\hline Phyla nodiflora (L.) Greene & Verbenaceae & TE,SL,WE,GR,DI & IV, VIII, XI, XII & 6.3 \\
\hline Alhagi graecorum Boiss. & Leguminosae & TE,SL,WE,DI & I, II, IV, VII, VIII & 5.2 \\
\hline Plantago major $\mathrm{L}$. & Plantaginaceae & TE,WE,GR,DI,AG & VII, VIII, XI, XII & 3.0 \\
\hline Lotus glaber Mill. & Leguminosae & GR & XI, XII & 2.6 \\
\hline $\begin{array}{l}\text { Silybum marianum var. marianum (L.) } \\
\text { Gaertn. }\end{array}$ & Compositae & TE,SL,WE & VII, VIII & 1.5 \\
\hline Oxalis corymbosa DC. & Oxalidaceae & DI,AG & VIII & 0.4 \\
\hline Spergularia marina (L.) Griesb. & Caryophyllaceae & TE,SL,WE,DI & VII & 0.4 \\
\hline $\begin{array}{l}\text { Geophytes-Helophytes } \\
\text { Phragmites australis (Cav.) Trin. Ex } \\
\text { Steud. }\end{array}$ & Gramineae & TE,SL,WE,OW,GR,DI & All except III, V, IX, X & 38.4 \\
\hline Vossia cuspidata (Roxb.) Griff. & Gramineae & TE,SL,WE,OW,DI & $\begin{array}{l}\text { I, V, VI, VII, VIII, IX, } \\
\text { XI }\end{array}$ & 34.7 \\
\hline Cynodon dactylon (L.) Pers. & Gramineae & TE,SL,WE,GR,DI,AG & $\begin{array}{l}\text { IV, VII, VIII, XI, XII, } \\
\text { XIII }\end{array}$ & 34.3 \\
\hline Echinochloa stagnina (Retz.) P. Beauv. & Gramineae & TE,SL,WE,OW,DI & IV, VI, VII, VIII, XI & 31.7 \\
\hline
\end{tabular}


Vegetation-environment relationships in south Nile Delta

\begin{tabular}{|c|c|c|c|c|}
\hline Persicaria senegalensis (Meisn) Sojak & Polygonaceae & TE,SL,WE,OW,DI & $\begin{array}{l}\text { I, II, V, VI, VII, VIII, } \\
\text { XIII }\end{array}$ & 17.3 \\
\hline Cyperus alopecuroides $\mathrm{L}$. & Cyperaceae & TE,SL,WE,OW,DI & V, VI, VII, VIII, XI & 16.2 \\
\hline Cyperus rotundus $\mathrm{L}$. & Cyperaceae & TE,SL,WE,GR,DI,AG & VII, VIII, XI, XIII & 14.4 \\
\hline Persicaria salicifolia (Willd.) Assenov & Polygonaceae & TE,SL,WE,OW,DI & VI, VII, VIII, XIII & 10.3 \\
\hline Oxalis corniculatus $\mathrm{L}$. & Oxalidaceae & GR,DI,AG & IV, VIII, XI, XII & 9.6 \\
\hline Paspalum dilatatum Poir. & Gramineae & GR,AG & XII & 7.7 \\
\hline Imperata cylindrica (L.) Raeusch. & Gramineae & TE,SL,WE,GR,DI,AG & I, IV, VII, VIII, XI, XII & 7.4 \\
\hline Mentha longifolia (L.) Huds. & Labiatae & TE,SL,WE,DI & IV, VIII, XII & 5.9 \\
\hline Panicum repens $\mathrm{L}$. & Gramineae & TE,SL,WE,GR,DI,AG & $\begin{array}{l}\text { IV, VII, VIII, XI, XII, } \\
\text { XIII }\end{array}$ & 4.4 \\
\hline Arundo donax $\mathrm{L}$. & Gramineae & TE,SL,WE,OW & VII, VIII & 3.7 \\
\hline Cyperus articulatus $\mathrm{L}$. & Cyperaceae & TE,SL,WE, DI & VII, VIII, XIII & 3.7 \\
\hline Paspalidium geminatum (Forssk.) Stapf & Gramineae & SL,WE,OW,GR,DI,AG & I, VII, VIII & 3.7 \\
\hline Persicaria lapathifolia (L.) Gray & Polygonaceae & TE,SL,WE & VII, VIII & 3.7 \\
\hline $\begin{array}{l}\text { Typha domingensis (Pers.) Poir. ex } \\
\text { Steud. }\end{array}$ & Typhaceae & SL,WE,OW & VIII & 3.7 \\
\hline Paspalum distichum $\mathrm{L}$. & Gramineae & TE,SL,WE,DI & VII, VIII, XII, XIII & 3.0 \\
\hline Desmostachya bipinnata (L.) Stapf. & Gramineae & TE,SL,WE & I, IV, VII, VIII & 2.2 \\
\hline Commicarpus helenae (Schult.) Meikle & Nyctaginaceae & GR & XII & 1.5 \\
\hline Cyperus longus $\mathrm{L}$. & Cyperaceae & TE,SL,WE,GR, ,AG & VII, XI & 0.7 \\
\hline Leptochloa fusca (L.) Kunth & Gramineae & WE,DI,AG & VIII, $\mathrm{X}$ & 0.7 \\
\hline Veronica anagallis-aquatica $\mathrm{L}$. & Scrophulariaceae & SL,WE,DI & XII & 0.7 \\
\hline Veronica persica Poir. & Scrophulariaceae & SL,GR,DI & XI, XII & 0.7 \\
\hline Allium roseum $\mathrm{L}$. & Alliaceae & GR & XI & 0.4 \\
\hline Alternanthera sessilis (L.) DC. & Amaranthaceae & SL,WE,DI & II & 0.4 \\
\hline Mentha piperita $\mathrm{L}$. & Labiatae & GR & XI & 0.4 \\
\hline Persicaria lanigera (R.Br.) Sojak & Polygonaceae & WE & VII & 0.4 \\
\hline Cyperus papyrus $\mathrm{L}$. & Cyperaceae & WE & III & 0.4 \\
\hline \multicolumn{5}{|l|}{ Hydrophytes } \\
\hline Eichhornia crassipes (C. Mart.) Solms & Pontederiaceae & SL,WE,OW & V, VI, VII, VIII, XIII & 14.0 \\
\hline $\begin{array}{l}\text { Ludwigia stolonifera (Guill. \& Perr.) } \\
\text { P.H. Raven }\end{array}$ & Onagraceae & SL,WE,OW & V, VI, VII, VIII, XI & 10.0 \\
\hline Myriophyllum spicatum L. & Haloragidaceae & WE,OW & V, VI, VII & 7.0 \\
\hline Lemna gibba $\mathrm{L}$. & Lemnaceae & OW & VIII & 1.8 \\
\hline Potamogeton nodosus Poir. & Potamogetonaceae & WE,OW & VI, VII, XI & 1.8 \\
\hline Ceratophyllum demersum $\mathrm{L}$. & Ceratophyllaceae & OW & V, VI, VII & 1.1 \\
\hline Potamogeton pectinatus $\mathrm{L}$. & Potamogetonaceae & OW & VI, XI & 0.7 \\
\hline Potamogeton crispus L. & Potamogetonaceae & OW & V & 0.4 \\
\hline \multicolumn{5}{|l|}{ Parasites } \\
\hline Orobanche crenata Loefl. & Orobanchaceae & $\mathrm{AG}$ & XI & 0.7 \\
\hline Cuscuta pedicellata Ledeb. & Convolvulaceae & WE,GA,DI,AG & VIII & 0.4 \\
\hline
\end{tabular}


Table 4. Unique species recorded in only one of the 7 habitats identified in south Nile Delta. $\mathrm{TE}=$ terraces, $\mathrm{SL}=$ slopes, $\mathrm{WE}=$ water edge, $\mathrm{OW}=$ open water, GR $=$ gardens $\&$ nurseries, $\mathrm{DI}=$ ditches, fallow $\&$ flooded lands and $\mathrm{AG}=$ croplands.

\begin{tabular}{|c|c|c|c|c|c|c|c|}
\hline \multirow{2}{*}{ Species } & \multicolumn{7}{|c|}{ Habitat type } \\
\hline & TE & SL & $\mathbf{W E}$ & OW & $\overline{G R}$ & DI & $\mathbf{A G}$ \\
\hline Enarthrocarpus lyratus & + & & & & & & \\
\hline Tamarix nilotica & + & & & & & & \\
\hline Trigonella hamosa & + & & & & & & \\
\hline Ceruana pratensis & & + & & & & & \\
\hline Lantana camara & & & + & & & & \\
\hline Persicaria lanigera & & & + & & & & \\
\hline Lemna gibba & & & & + & & & \\
\hline Potamogeton crispus & & & & + & & & \\
\hline Potamogeton nodosus & & & & + & & & \\
\hline Potamogeton pectinatus & & & & + & & & \\
\hline Allium roseum & & & & & + & & \\
\hline Commicarpus helenae & & & & & + & & \\
\hline Eragrostis cilianensis & & & & & + & & \\
\hline Lotus glaber & & & & & + & & \\
\hline Mentha piperita & & & & & + & & \\
\hline Paspalum dilatatum & & & & & + & & \\
\hline Polygonum equisetiforme & & & & & + & & \\
\hline Abutilon theophrasti & & & & & & + & \\
\hline Bergia capensis & & & & & & + & \\
\hline Cyperus difformis & & & & & & + & \\
\hline Cyperus papyrus & & & & & & + & \\
\hline Fimbristylis bisumbellata & & & & & & + & \\
\hline Gynandropsis gynandra & & & & & & + & \\
\hline Juncus bufonius & & & & & & + & \\
\hline Amaranthus spinosus & & & & & & & + \\
\hline Cenchrus echinatus & & & & & & & + \\
\hline Coronopus squamatus & & & & & & & + \\
\hline Eragrostis pilosa & & & & & & & + \\
\hline Euphorbia indica & & & & & & & + \\
\hline Malvastrum coromandelianum & & & & & & & + \\
\hline Orobanche crenata & & & & & & & + \\
\hline Setaria italica & & & & & & & + \\
\hline Total & 3 & 1 & 2 & 4 & 7 & 7 & 8 \\
\hline
\end{tabular}




\section{(a) Agglomerative clustering}

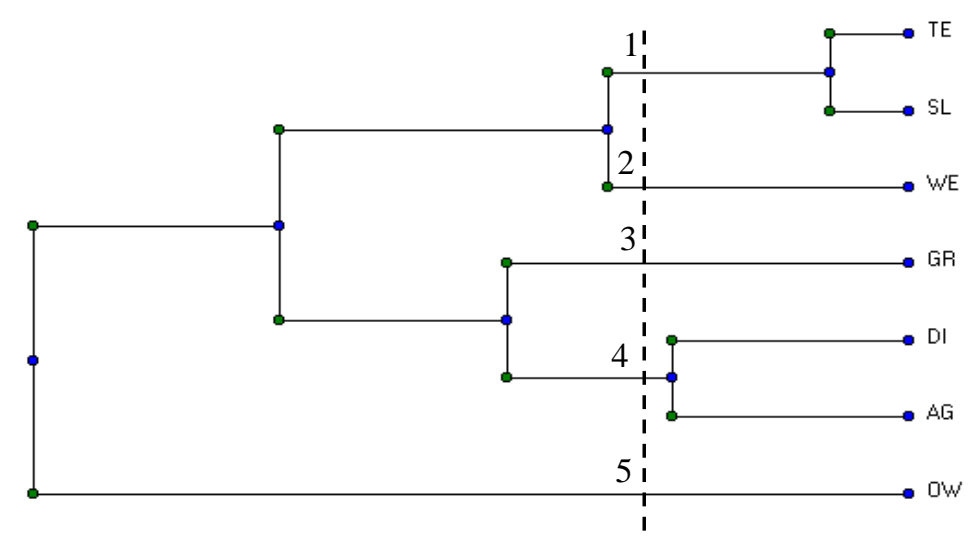

(b) Polar ordination

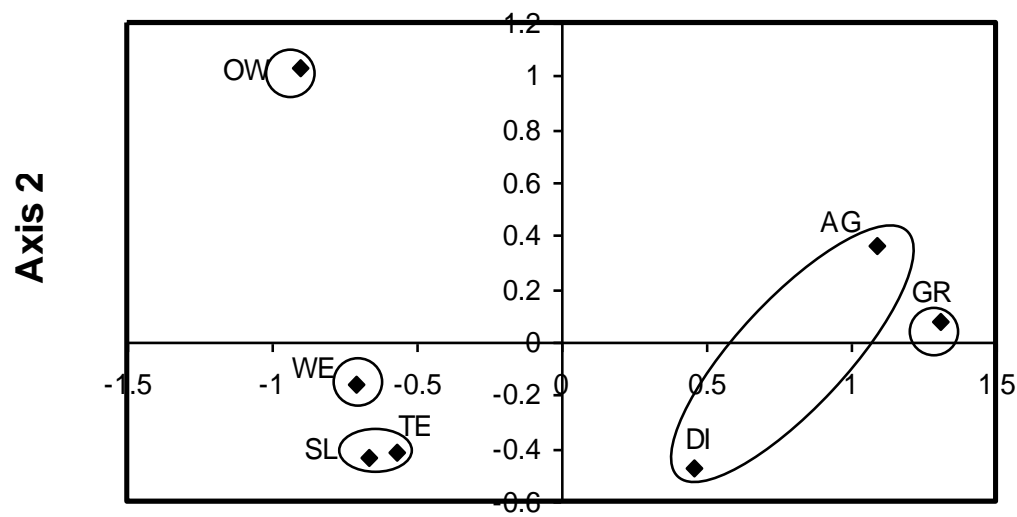

Axis 1

Fig. 2. The dendrogram resulting from the agglomerative clustering technique (a), and the similarity ordination according to Sørensen 1948 (b) of the plant communities of the 7 habitats in south Nile Delta. The habitats are: $\mathrm{TE}=$ terraces, $\mathrm{SL}=$ slopes, $\mathrm{WE}=$ water edge, $\mathrm{OW}=$ open water, GR = gardens \& nurseries, DI = ditches, fallow lands \& flooded lands and $\mathrm{AG}=$ croplands. 
Table 5. Life forms of the recorded species in the 7 habitats in south Nile Delta. A: actual number of species, R: relative number of species, $\mathrm{TE}=$ terraces, $\mathrm{SL}=$ slopes, $\mathrm{WE}=$ water edge, $\mathrm{OW}=$ open water, $\mathrm{GR}=$ gardens \& nurseries, DI = ditches, fallow \& flooded lands and $A G=$ croplands. The maximum and minimum values are underlined.

\begin{tabular}{|c|c|c|c|c|c|c|c|c|c|}
\hline \multirow{2}{*}{ Life form } & & \multicolumn{7}{|c|}{ Habitat } & \multirow{2}{*}{$\begin{array}{c}\text { Total } \\
\text { species }\end{array}$} \\
\hline & & TE & SL & WE & OW & GR & DI & AG & \\
\hline \multirow{2}{*}{ Phanerophytes } & A & 4 & 3 & 4 & 3 & 1 & 2 & 1 & 5 \\
\hline & $\mathrm{R}$ & 5.6 & 4 & 4.8 & 12.5 & 1.3 & 2.1 & 1.3 & 3.4 \\
\hline \multirow[t]{2}{*}{ Chamaephytes } & A & 6 & 6 & 6 & 1 & 4 & 6 & 2 & 7 \\
\hline & $\mathrm{R}$ & 8.4 & 8.1 & 7.1 & 4.1 & 5.4 & 6.2 & 2.6 & 4.8 \\
\hline \multirow[t]{2}{*}{ Hemicryptophytes } & A & $\overline{6}$ & 6 & 6 & $\overline{---}$ & 6 & 5 & 3 & 8 \\
\hline & $\mathrm{R}$ & $\underline{8.4}$ & 8.1 & 7.1 & --- & 8 & 5.2 & $\underline{3.8}$ & 5.5 \\
\hline \multirow[t]{2}{*}{ Geophytes -heleophytes } & A & $\overline{15}$ & 19 & 19 & 9 & 13 & 19 & $\overline{7}$ & 30 \\
\hline & $\mathrm{R}$ & 21.1 & 25.7 & 22.6 & $\underline{37.5}$ & 17.5 & 19.6 & $\underline{9}$ & 20.8 \\
\hline \multirow[t]{2}{*}{ Hydrophytes } & A & 1 & 2 & 4 & 8 & --- & --- & --- & 8 \\
\hline & $\mathrm{R}$ & 1.4 & 2.7 & 4.7 & 33.3 & --- & --- & --- & 5.5 \\
\hline \multirow[t]{2}{*}{ Therophytes } & A & $\overline{39}$ & 38 & 45 & $\overline{7}$ & 50 & 65 & 63 & 84 \\
\hline & $\mathrm{R}$ & 54.9 & 51.3 & 53.6 & $\underline{29.1}$ & 67.6 & 67 & $\underline{80.8}$ & $\underline{58.3}$ \\
\hline \multirow[t]{2}{*}{ Parasites } & A & --- & --- & --- & $\overline{---}$ & 1 & 1 & $\overline{2}$ & $\overline{2}$ \\
\hline & $\mathrm{R}$ & --- & --- & --- & --- & 1.3 & $\underline{1}$ & 2.6 & 1.4 \\
\hline Total & & 71 & 74 & 84 & 24 & 74 & 97 & $\overline{78}$ & 144 \\
\hline
\end{tabular}

Table 6. Seasonal variation in the recorded species in the 7 habitats in the south Nile Delta.

\begin{tabular}{|l|cccc|c|}
\hline Habitat & Spring & Summer & Autumn & Winter & $\begin{array}{c}\text { All the } \\
\text { year }\end{array}$ \\
& & & & & \\
\hline Terraces & 48 & 44 & 45 & 43 & 71 \\
Slopes & 53 & 49 & 46 & 50 & 74 \\
Water edges & 57 & 46 & 52 & 58 & 84 \\
Open water & 18 & 15 & 16 & 21 & 24 \\
Gardens \& nurseries & 61 & 38 & 39 & 38 & 74 \\
Ditches, fallow \& flooded lands & 71 & 60 & 48 & 53 & 97 \\
Croplands & 56 & 39 & 41 & 45 & 78 \\
\hline Total species per season & 123 & 106 & 98 & 103 & 144 \\
\hline
\end{tabular}


(a)

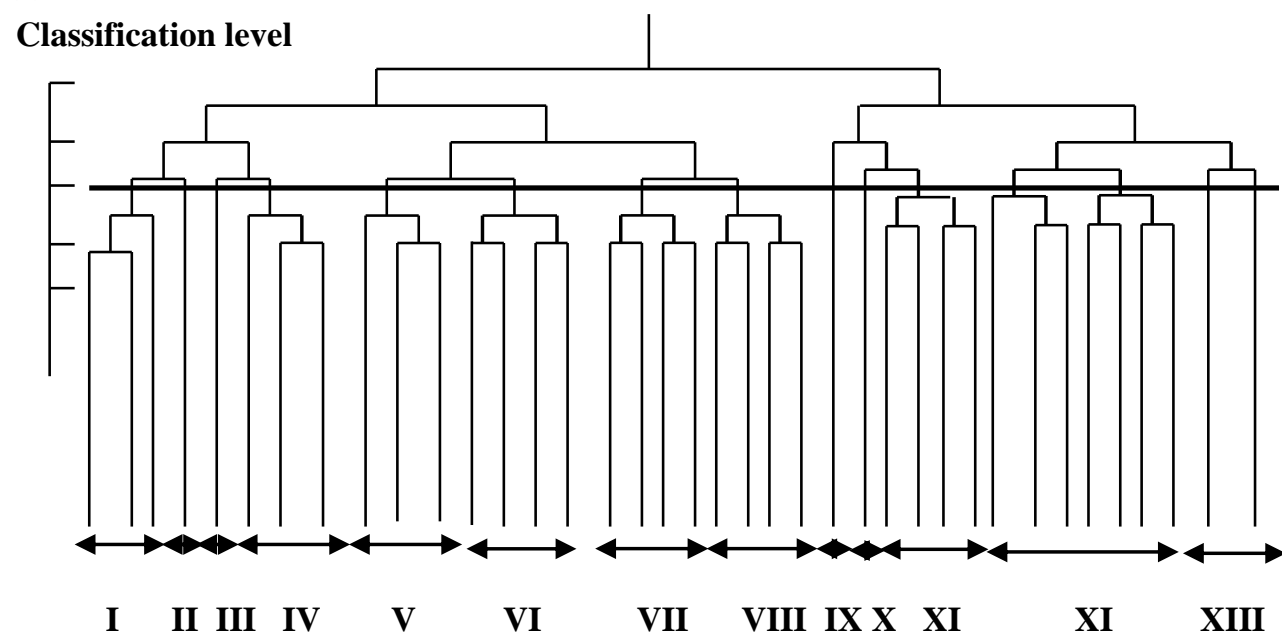

(b)

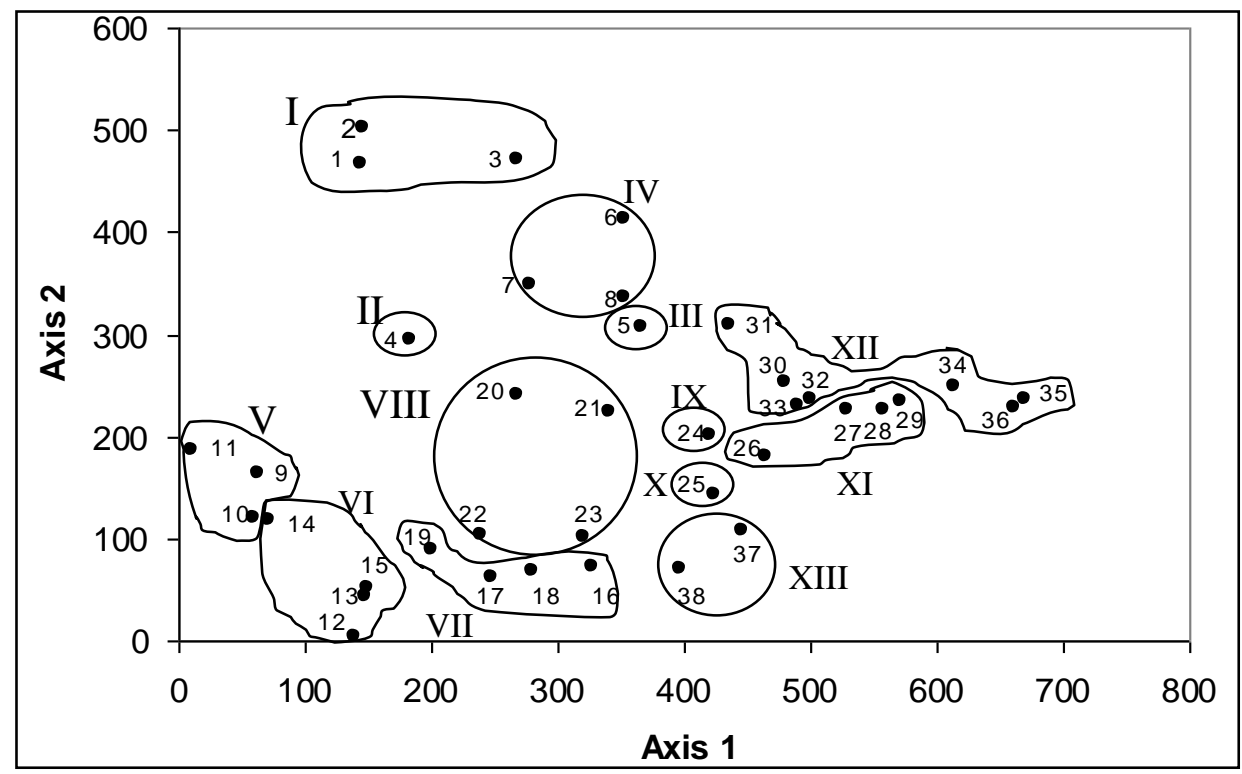

Fig. 3. The relationship between the 13 groups generated after the application of TWINSPAN classification technique on 271 sampled stands in the different habitats (a), and the position of their cluster centroids on the first and second axes of DCA. 


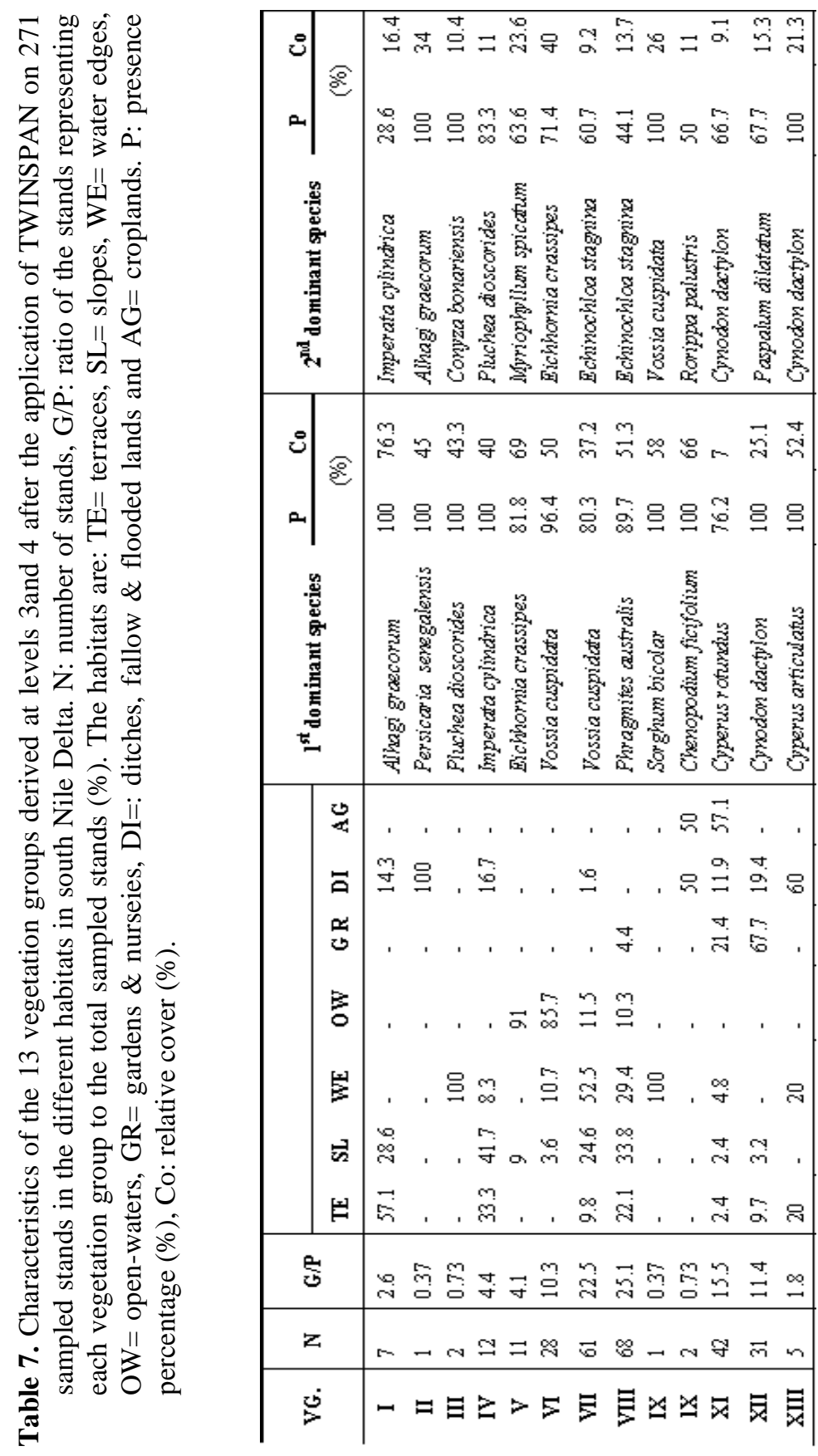


Table 8. Variation in some diversity indices calculated for the 13 vegetation groups derived after the application of TWINSPAN on 271 stands in the different habitats in south Nile Delta. The maximum and minimum values are underlined.

\begin{tabular}{|c|ccccc|}
\hline VG & $\begin{array}{c}\text { Total } \\
\text { species }\end{array}$ & $\begin{array}{c}\text { Species } \\
\text { richness }\end{array}$ & $\begin{array}{c}\text { Species } \\
\text { turnover }\end{array}$ & $\begin{array}{c}\text { Relative conc. } \\
\text { of dominance }\end{array}$ & $\begin{array}{c}\text { Relative } \\
\text { evenness }\end{array}$ \\
\hline I & 10 & 2.4 & 4.2 & 6.2 & 2 \\
II & 7 & 7 & $\underline{1}$ & 7 & 2 \\
III & 8 & 7 & 1.1 & 22.5 & 2 \\
IV & 22 & 5.3 & 4.1 & 12.1 & 2.7 \\
V & 8 & $\underline{2.2}$ & 3.6 & 4 & 1.6 \\
VI & 14 & 4 & 3.7 & 7.1 & 2.1 \\
VII & 55 & 6.6 & 8.3 & 21 & 3.4 \\
VIII & 64 & 5 & $\underline{13.1}$ & 17.5 & 3.4 \\
IX & $\underline{3}$ & 3 & 1 & $\underline{3}$ & $\underline{1.1}$ \\
$\mathbf{1 X}$ & 8 & 4.5 & 2 & 36 & 2 \\
XI & $\underline{87}$ & $\underline{11.6}$ & 7.5 & $\underline{37.4}$ & $\underline{4}$ \\
$\mathbf{X I I}$ & 54 & 7.7 & 7 & 23 & 3.5 \\
$\mathbf{X I I I}$ & 16 & 6 & 2.7 & 15.5 & 2.6 \\
\hline Mean & 27.4 & 5.6 & 4.6 & 16.3 & 2.5 \\
\hline
\end{tabular}

\section{Discussion}

Comparing the recorded species in the present study (144 species) with those recorded in the north and middle Delta, it was found that $61.8 \%$ of them were recorded in the study of Al-Sodany (1998) along the watercourses of north Nile Delta, and $45.4 \%$ in the study of El-Sheikh (1996) in the ruderal habitats of the Nile Delta. On the contrary, all the halophytic and desert plants in the north Delta were not recorded in the present study. Moreover, some aquatic plants that were recorded in the study of Al-Sodany (1998) were not recorded in the present study (e.g. 

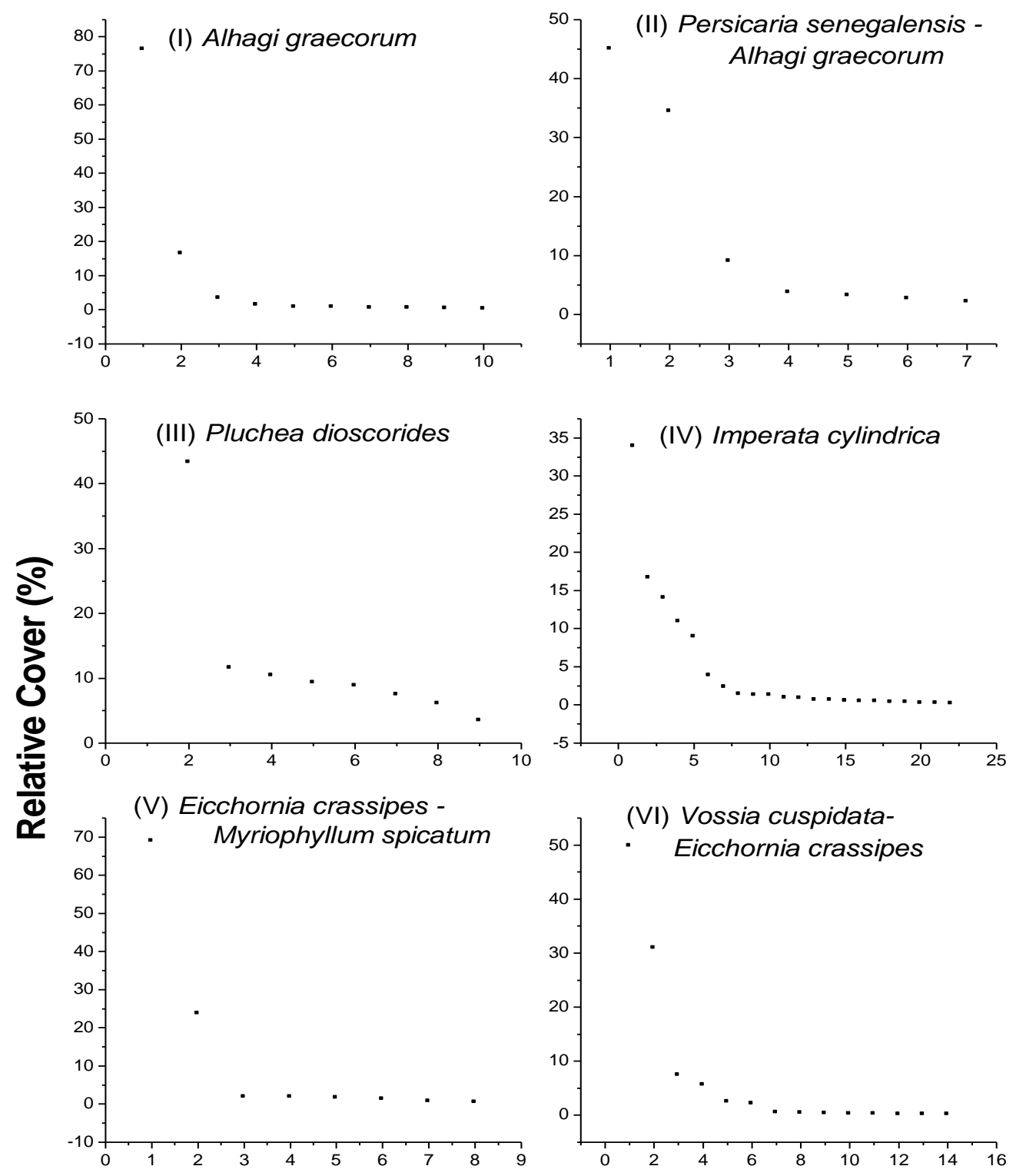

\section{Species Sequence}

Fig. 4. Dominance diversity curves of the 13 vegetation groups identified in south Nile Delta $(1-13)$. 
Fig. 4. Continue
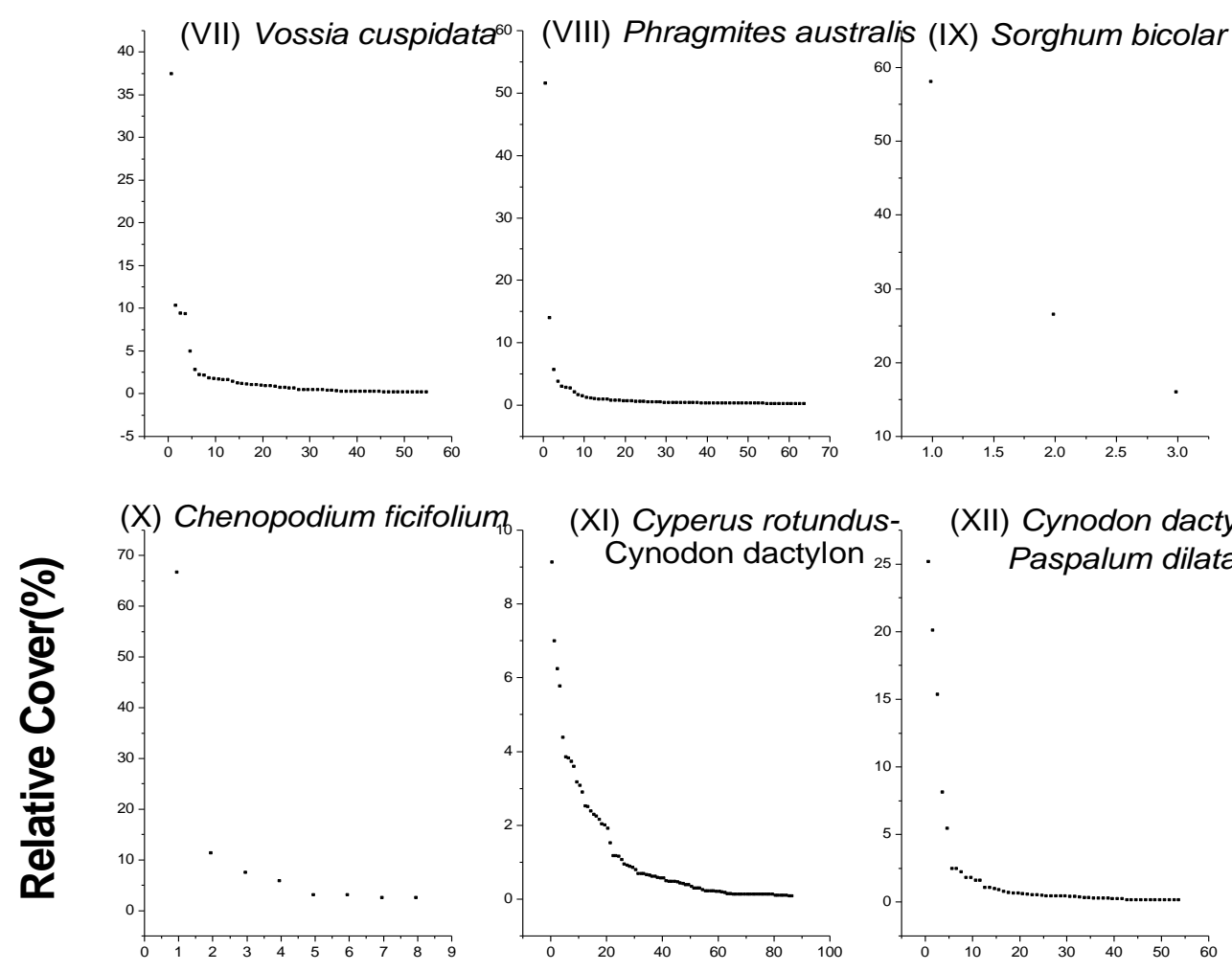

(XII) Cynodon dactylon-
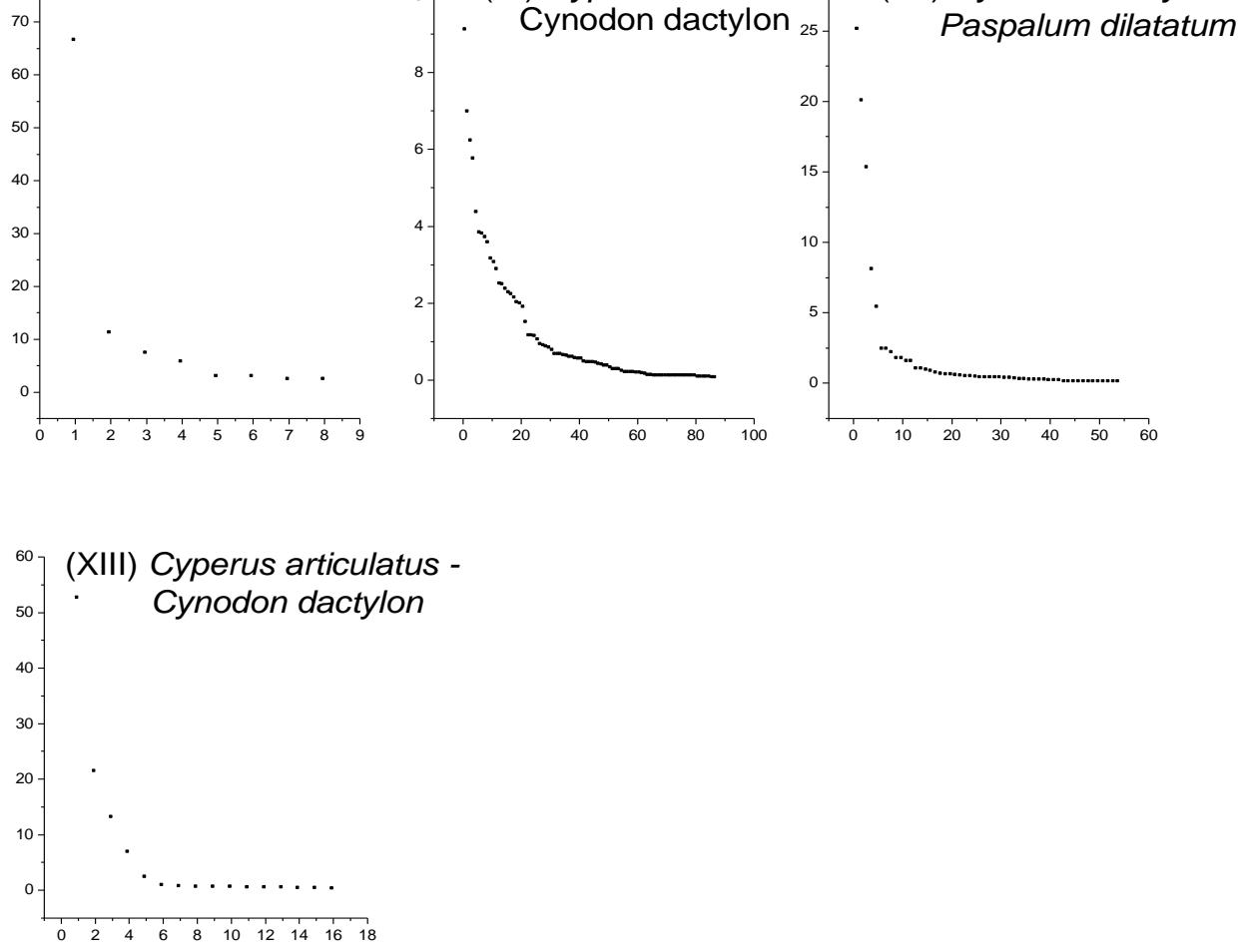
Table 9. Mean ( \pm standard deviation) of the soil characteristics of the 13 vegetation groups generated after the application of TWINSPAN. The maximum and minimum values are underlined. The F-values and its probability are indicated. $* \mathrm{p} \leq 0.05, * * \mathrm{p} \leq 0.01, * * * \mathrm{p} \leq 0.001$.

\begin{tabular}{|c|c|c|c|c|c|c|c|c|c|c|c|c|c|c|}
\hline \multirow{2}{*}{\multicolumn{2}{|c|}{$\begin{array}{l}\text { Soil } \\
\text { characteristics }\end{array}$}} & \multicolumn{11}{|c|}{ Vegetation group } & \multirow{2}{*}{ Mean \pm SD } & \multirow{2}{*}{ F-value } \\
\hline & & I & II & IV & V & VI & VII & VIII & $\mathbf{X}$ & XI & XII & XIII & & \\
\hline Sand & & 83.0 & 91.9 & 80.4 & 86.7 & 76.7 & 88.7 & 78.4 & 79.4 & $\underline{76.0}$ & 79.1 & 91.0 & $82.8 \pm 5.8$ & $2.8^{* *}$ \\
\hline Silt & $\%$ & 10.3 & $\underline{4.0}$ & 14.9 & 6.0 & 10 & 6.9 & 16.6 & 14.0 & $\underline{19.2}$ & 15.7 & 4.0 & $11.1 \pm 5.3$ & $1.7 * *$ \\
\hline Clay & & 6.7 & $\underline{4.1}$ & 4.7 & 7.3 & $\underline{13.3}$ & 4.4 & 5.0 & 6.6 & 4.9 & 5.2 & 5.1 & $6.1 \pm 2.6$ & 1.6 \\
\hline \multicolumn{2}{|l|}{ pH } & 7.3 & $\underline{7.9}$ & 7.7 & 7.9 & $\underline{6.8}$ & 7.7 & 7.8 & 7.6 & 7.8 & 7.8 & 7.7 & $7.6 \pm 0.3$ & $2.5^{* *}$ \\
\hline \multicolumn{2}{|c|}{ E.C $\left(\mathrm{mS} \mathrm{cm}^{-1}\right)$} & $\underline{5.2}$ & 5.2 & 3.1 & 1.7 & $\underline{2}$ & 2.1 & 2.3 & 0.3 & 0.9 & 1.3 & $\underline{0.3}$ & $2.2 \pm 1.7$ & $4.5^{* * * *}$ \\
\hline $\mathrm{HCO}_{3}$ & \multirow{10}{*}{$\begin{array}{l}760 \\
90 \\
=\end{array}$} & 0.2 & $\underline{0.1}$ & 0.2 & 0.1 & $\underline{0.4}$ & 0.1 & 0.1 & 0.1 & 0.1 & 0.1 & 0.1 & $0.14 \pm 0.09$ & $3.3 * * *$ \\
\hline $\mathrm{Cl}$ & & 7 & 7 & 7 & 2 & 2.5 & 2 & 3 & $\underline{0.4}$ & 1 & 1 & 0.5 & $3.04 \pm 2.7$ & $3.6 * * *$ \\
\hline $\mathrm{SO}_{4}$ & & 0.2 & 0.4 & 0.2 & 0.1 & 0.9 & 0.2 & $\underline{4}$ & 0.2 & 0.2 & 0.2 & $\underline{0.1}$ & $0.61 \pm 1.15$ & $1.1 * * *$ \\
\hline $\mathrm{NO}_{3}$ & & $\underline{6}$ & $\underline{0.0}$ & 5 & 2 & 2 & 2 & 3 & 1 & 3 & 2 & 3 & $2.63 \pm 1.7$ & 5.4 \\
\hline $\mathrm{NO}_{2}$ & & 223 & 44 & 251 & 111 & $\underline{552}$ & 105 & 132 & 100 & $\underline{64}$ & 104 & 64 & $\begin{array}{c}159.1 \pm \\
145.2\end{array}$ & $2.3 * * *$ \\
\hline $\mathbf{P}$ & & 232 & $\underline{387}$ & 245 & 90 & 159 & 134 & 144 & $\underline{89}$ & 151 & 172 & 122 & $175 \pm 86.0$ & $6.0^{*}$ \\
\hline $\mathrm{Na}$ & & 9 & 9 & 5 & 2 & $\underline{37}$ & 3 & 4 & 1 & 1 & 2 & $\underline{0.2}$ & $6.65 \pm 10.5$ & $1.2^{* * * *}$ \\
\hline $\mathbf{K}$ & & 0.02 & $\underline{0.03}$ & 0.02 & $\underline{0.01}$ & 0.03 & 0.02 & 0.02 & 0.02 & 0.02 & 0.02 & 0.01 & $\begin{array}{c}0.02 \pm \\
0.006\end{array}$ & 5.2 \\
\hline $\mathrm{Ca}$ & & 1 & 2 & 1 & 1 & $\underline{3}$ & 1 & 1 & 1 & 1 & 1 & $\underline{0.0}$ & $1.2 \pm 0.75$ & $6.7 * * *$ \\
\hline Mg & & 1 & 1 & 1 & 1 & $\underline{3}$ & 1 & 1 & 1 & 1 & 1 & $\underline{0.0}$ & $1.1 \pm 0.7$ & $0.0 * * *$ \\
\hline \multicolumn{2}{|c|}{$\mathrm{CaCO}_{3}(\%)$} & 5.0 & 2.1 & 3.3 & 3.8 & $\underline{6.8}$ & 2.5 & 2.5 & 2.6 & 2.8 & 2.6 & 1.7 & $\underline{3.2 \pm 1.5}$ & $2.3^{* * *}$ \\
\hline
\end{tabular}

Nymphaea coerulea, Ottelia alismoides, and Najas armata). Eighteen species $(12.5 \%)$ recorded in the present study were not recorded neither by Al-Sodany (1998) nor by El-Sheikh (1989): e.g. Vossia cuspidata, Persicaria lanigera, Commicarpus helenae, Malvastrum coromandelianum, Amaranthus spinosus, Homognaphalium pulvinatum, Paspalum dilatatum, Oxalis corymbosa and Rorippa palustris. In addition, most of these species were not recorded in the study of Hussein (2000) in the greater Cairo (southern border of the study area). 
Table 10. Mean ( \pm standard deviation) of water characteristics of the 13 vegetation groups generated after the application of TWINSPAN. The maximum and minimum values are underlined. F-values and its probability are indicated.

\begin{tabular}{|c|c|c|c|c|c|c|c|c|c|c|c|}
\hline \multirow{2}{*}{\multicolumn{2}{|c|}{$\begin{array}{c}\text { Water } \\
\text { characteristics }\end{array}$}} & \multicolumn{8}{|c|}{ Vegetation group } & \multirow{2}{*}{ Mean \pm SD. } & \multirow{2}{*}{$\begin{array}{c}\text { F- } \\
\text { value }\end{array}$} \\
\hline & & III & IV & V & VI & VII & VIII & IX & XI & & \\
\hline \multicolumn{2}{|l|}{ pH } & 9.2 & $\underline{11.0}$ & 9.7 & 9.5 & 9.9 & 9.9 & 7.7 & 7.8 & $9.3 \pm 1.1$ & 1.7 \\
\hline \multicolumn{2}{|c|}{ E.C $\left(\mathrm{mS} \mathrm{cm}^{-1}\right)$} & 0.3 & 0.3 & 0.3 & 0.3 & 0.3 & $\underline{0.9}$ & 0.3 & 0.3 & $0.4 \pm 0.2$ & $5 * * *$ \\
\hline $\mathrm{HCO}_{3}$ & \multirow{11}{*}{$\operatorname{Mg~l}^{-1}$} & 0.2 & 0.2 & 0.2 & 0.1 & 0.2 & $\underline{0.4}$ & 0.2 & 0.2 & $0.2 \pm 0.08$ & $4.4 * * *$ \\
\hline $\mathrm{Cl}$ & & 0.02 & 0.02 & $\underline{0.002}$ & $\begin{array}{c}0 \\
0.02\end{array}$ & 0.01 & $\underline{0.1}$ & 0.03 & 0.02 & $0.03 \pm 0.03$ & $5.5 * * *$ \\
\hline $\mathrm{SO}_{4}$ & & 16.2 & $\underline{14.1}$ & 19.5 & 18.1 & 18.8 & 24.7 & $\underline{28.3}$ & 16.4 & $19.5 \pm 4.73$ & $3.5 * * *$ \\
\hline $\mathrm{NO}_{3}$ & & 0.1 & 0.1 & 0.1 & 0.1 & 0.1 & 0.1 & 0.1 & 0.1 & $0.1 \pm 0.0$ & 1.8 \\
\hline $\mathrm{NO}_{2}$ & & $\underline{0.4}$ & 0.4 & $\underline{0.6}$ & 0.5 & 0.5 & 0.5 & 0.4 & 0.6 & $0.5 \pm 0.08$ & 1.1 \\
\hline $\mathbf{P}$ & & 13.4 & 13.6 & 1.4 & $\underline{13.4}$ & 13.6 & $\underline{2.0}$ & 13.9 & 13.6 & $10.6 \pm 5.5$ & $4 * * *$ \\
\hline $\mathbf{N a}$ & & 0.04 & 0.04 & 0.04 & 0.04 & 0.09 & $\underline{1.1}$ & 0.03 & 0.03 & $0.17 \pm 0.36$ & $4.5^{* * * *}$ \\
\hline $\mathbf{K}$ & & $\underline{0.003}$ & 0.003 & $\underline{0.004}$ & 0.01 & 0.005 & 0.01 & 0.003 & 0.02 & $0.007 \pm 0.36$ & 1.5 \\
\hline $\mathbf{C a}$ & & 1.1 & 1.4 & $\underline{1.0}$ & 1.2 & 1.2 & $\underline{2.7}$ & 1.4 & 1.0 & $1.4 \pm 0.006$ & $4.3 * * *$ \\
\hline Mg & & 0.4 & 0.4 & 0.4 & 0.4 & 0.4 & $\underline{1.2}$ & 0.6 & 0.3 & $0.51 \pm 0.56$ & $3.4^{* * * *}$ \\
\hline $\mathrm{CO}_{3}$ & & 0.01 & 0.0 & 0.03 & 0.03 & 0.03 & 0.02 & 0.03 & 0.03 & $0.02 \pm 0.03$ & 0.7 \\
\hline \multicolumn{2}{|c|}{$\begin{array}{l}\text { Dissolved } \\
\text { Oxygen (ppm) }\end{array}$} & $\underline{3.3}$ & 3.5 & 5.7 & 6.9 & 5.7 & 4.4 & 4.1 & $\underline{11.2}$ & $5.6 \pm 11.6$ & 0.5 \\
\hline
\end{tabular}

The life form spectrum indicated that therophytes were highly represented, followed by geophytes-helophytes and hydrophytes. This is in accordance with the study of Al-Sodany (1992) in North Delta and the general trend of Egyptian flora (Shaltout \& El-Halawany, 1993). Croplands, gardens and nurseries had the highest number of therophytes, while the open water had the lowest. This may be due to that therophytes are not adapted to grow in or above the water surface and inherently do not develop subterranean perennating organs which fix the plant to the bottom soil of the water body as in the case of perennial sedges and rushes (Al-Sodany, 1998). Geophytes-helophytes and hydrophytes were well represented along the slopes, water edges and open water more than the other habitats. This is due to the nature of these life forms, which often produce perennating organs to resist the external disturbances and impacts on the habitats or due to their water demands. Similar conclusions were reported by El-Demerdash (1984); 
Shaltout \& Sharaf El-Din (1988); El-Sheikh (1996) and Shaltout \& ElHalawany (1993) in their studies on the vegetation along the irrigation and drainage canals in Nile Delta.

Table 11. Simple linear correlation coefficient (r) between the pairs of soil and community variables.

\begin{tabular}{|l|l|c|c|}
\hline \multicolumn{2}{|c|}{ Pairs of variables } & r & P \\
\hline \multicolumn{2}{|l|}{ A- community variables } & 0.70 & 0.001 \\
\hline \multirow{2}{*}{ Total species } & x Species richness & 0.84 & 0.001 \\
& x Species turnover & 0.58 & 0.001 \\
& x Relative concentration of \\
& dominance & & 0.001 \\
\hline Relative evenness & x Relative evenness & 0.74 & 0.001 \\
& x Species richness & 0.79 & 0.001 \\
\hline CaCO ${ }_{3}$ & B- Community vs soil variables & -0.62 & 0.01 \\
\hline
\end{tabular}

The highest numbers of species per year were recorded in the ditches, fallow and flooded lands followed by the water edges and croplands. Winter and spring seasons were the flourishing seasons of the year in which most of the species were grown $(52.1 \%$ were winter active species and $85.4 \%$ were spring active species). This may reflects the effect of climatic factors and water stress on the seasonal existence of species. 


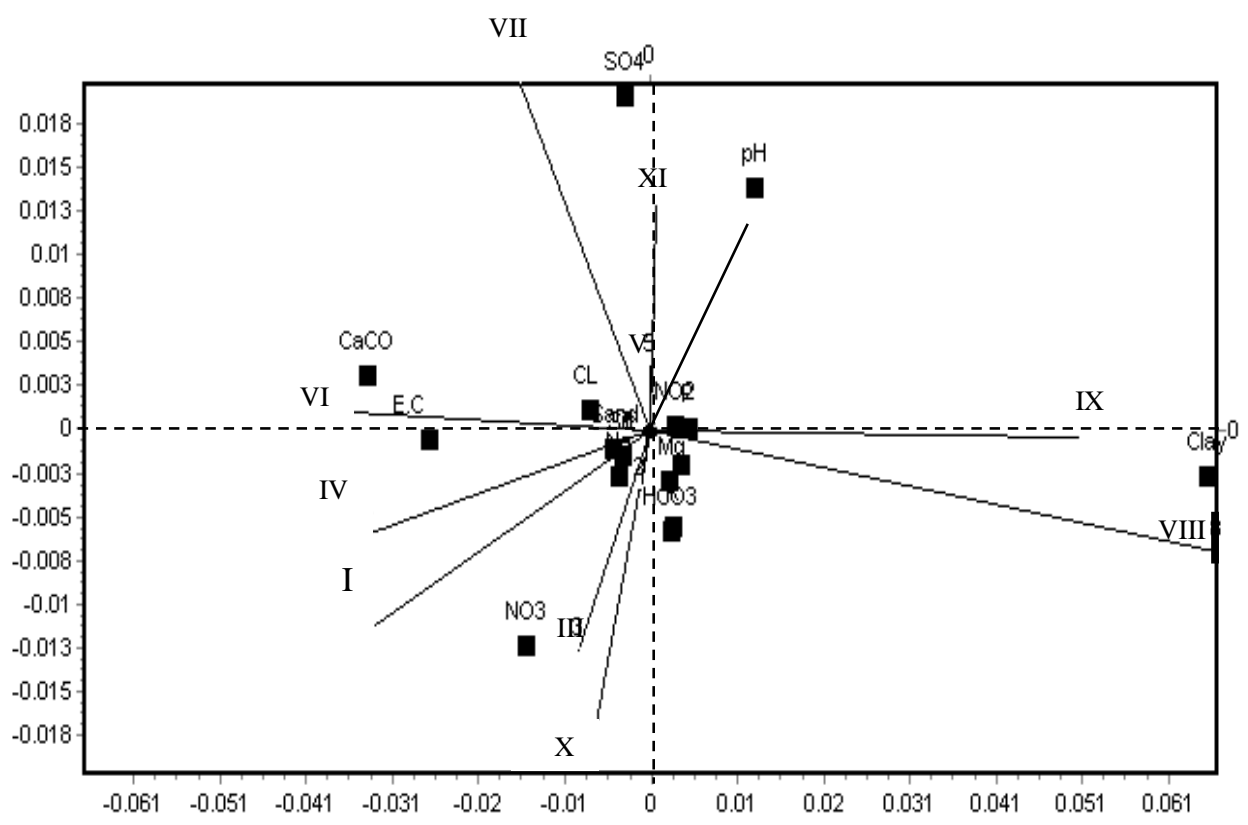

Fig. 5. PCA ordination diagram of the 13 vegetation groups generated after application of TWINSPAN (represented by numbers \& squares) with respect to soil variables (represented by lines).

According to TWINSPAN, thirteen vegetation groups were generated and their ordination using DCA represents a complex gradient of moisture started with the open water communities while the gardens and nurseries communities located at the opposite direction. Positions that represent the water edges were located between that of the open water in one side, and gardens and nurseries in the other side. The moisture gradients started with the plant communities inhabited the terraces and slope of the watercourses and subjected to heavy human impacts (Alhagi graecorum, Imperata cylindrica, and Pluchea dioscorides groups). The moisture gradient and human impacts increased gradually in the plant communities that colonized the ditches and flooded lands (e.g. Persicaria senegalensis-Alhagi graecorum, Chenopodium ficifolium, and Cyperus articulatus-Cynodon dactylon). Similar conclusions were reported by El-Sheikh (1996) and Al- 
Sodany (1998) in their studies on irrigation canals and drains in Middle and North Delta, respectively.

The mesophytes dominating plant communities in the gardens, nurseries and croplands include: Chenopodium ficifolium, Cynodon dactylonPaspalum dilatatum and Cyperus rotundus-Cynodon dactylon groups. Wetland plants that characterize Phragmites australis, Pluchea dioscorides, Sorghum bicolor and Cyperus articulatus-Cynodon dactylon groups, usually inhabited the edges of the watercourses. The moisture gradient ends with the plant communities of the open water (e.g. Eichhornia crassipesMyriophyllum spicatum, Vossia cuspidata and Vossia cuspidata-Eichhornia crassipes groups). The aquatic plants recorded in the present study have certain features in common, such as vegetative reproduction and relatively high rapid growth (Murphy et al., 1990). Other plants may tolerate physical disturbances by being strong and flexible (Spink, 1992). It was also observed that most of the plant communities, especially in terrestrial and aquatic habitats, were often overwhelmingly dominated by one species. The presence of plant community dominated by one species, results in the suppression of the less competitive species, and hence the decrease in the species diversity of that community (Mohler \& Liebman, 1987). Similar conclusions have been made by Shaltout et al. (1995) and Shaltout \& ElSheikh (2003) in their studies on the vegetation of the Mediterranean region of Nile Delta and that of the urban habitats in the Nile Delta region.

It is interesting to compare the aquatic plant communities in the present study ith those of the other related studies in the north (Al-Sodany, 1998) and middle Delta (El-Sheikh, 1989). Three vegetation groups have a common occurrence in north, middle and south Delta (Eichhornia crassipes, Echinochloa stagnina and Phragmites australis). Each region has its characteristic plant communities. Obviously, the present study area has its unique vegetation groups (e.g. Vossia cuspidata, Vossia cuspidataEichhornia crassipes and Eichhornia crassipes-Myriophyllum spicatum groups). Moreover, Ceratophyllum demersum-Potamogeton crispus, Potamogeton pectinatus and Azolla filliculoides were either completely absent from the study area or exist in a very low cover. It was reported by Fayed (1985), Springuel (1987) and Springuel \& Murphy (1990) that Myriophyllum spicatum invaded the Nile Valley in the southern provinces of Egypt. Northwards, in the Nile valley, El-Kholi (1989) recorded Myriophyllum spicatum in the River Nile and irrigation canals from Aswan to Giza, but he did not record it in the Nile Delta including the northern 
Lakes. In Nile Delta, Serag (1991); Zahran \& Willis (1992); Shaltout \& ElSheikh (1993) and Shaltout et al. (1994) did not refer to the presence of Myriophyllum spicatum. Recently, Khedr \& El-Demerdash (1995) and Khedr \& Zahran (1999) recorded Myriophyllum spicatum in the east of the Nile Delta and Lake Manzala (c.f. Zahran \& Willis 2003). Two vegetation groups of the present study (Cyperus rotundus-Cynodon dactylon and Cynodon dactylon-Paspalum dilatatum) were represented with their first dominant species in the study of El-Sheikh (2004) in El-Qanatir Public Park. A total of 67 species was recorded as weed vegetation in the study of El-Sheikh (2004) (74 species in the present study).

The lowest values of diversity indices were that of the plant communities of the open water. Some of the plant communities that occupy the gardens, nurseries, croplands, ditches, fallow and flooded lands had high diversity indices (e.g. Cynodon dactylon-Cyperus rotundus and Cynodon dactylon). In general, it was noted that the diversity indices of the plant communities in south Nile Delta were lower than that in North Delta and middle Delta (see Al-Sodany, 1998 and Shaltout et al. 1994). Steep dominance-diversity curves (geometric series) showed strong dominance of some species and confirm the hypothesis of niche-pre-emption, which often exhibited by vascular plants with a low diversity (Whittaker, 1972 and AlSodany, 1998). As an example for the strong dominance of species in the water edge and the open water communities were Pluchea dioscorides, Vossia cuspidata and Phragmites australis. Moreover, There was a strong dominance of Alhagi graecorum and Imperata cylindrica along the terraces and slopes of watercourses, and Chenopodium ficifolium in the croplands, ditches, fallow and flooded lands. Some plant communities were dominated by two species while the other species were weakly represented such as: Eichhornia crassipes-Myriophyllum spicatum, Vossia cuspidataEichhornia crassipes (in the open water) and Cynodon dactylon-Paspalum dilatatum (in the gardens and nurseries).

Principal Components Analysis revealed a degree of correlation between some soil variables and plant communities. It indicated a high correlation between Vossia cuspidata (VG VII) and Cyperus rotundus-Cynodon dactylon (VG XI) with the soil pH and sulphate contents. Shehata (1994) reported that Vossia cuspidata dominated in water with low alkaline $\mathrm{pH}$ (78.9), total soluble salts between 2.12-5.9 $\mathrm{mg} \mathrm{L}^{-1}$ and sulphates between 0.7$1.16 \mathrm{mg} \mathrm{L}^{-1}$. This may explain the vigorous and weak growth of the plant in fresh and saline water, respectively. It also revealed that Phragmites 
australis (VG VIII) and Sorghum bicolar (VG IX) were positively correlated with clay content. This explains the abundance of the two species along the water edges as well as in all the watercourse habitats.

\section{References}

Ahmed, D.A.A. 2003. Current situation of the flora and vegetation of the Nile Delta region. M.Sc. Thesis, Faculty of Science, Tanta University, Tanta. $321 \mathrm{pp}$.

Allen, S.E., Grimshaw, H.M., Parkinson, J.A. \& Quarmby, C. 1974. Chemical Analysis of Ecological Materials. Blackwell Scientific Publications, Oxford. $565 \mathrm{pp}$.

Al-Sodany, Y.M. 1992. Vegetation analysis of thenorthern part of Nile Delta region. M.Sc. Thesis, Faculty of Science, Tanta University, Tanta. 122 pp.

1998. Vegetation analysis of the canals, drains and lakes of the northern part of Nile Delta. Ph.D. Thesis, Faculty of Science, Tanta University, Tanta. 232pp.

Anonymous 1980. Climatic Normals of the Arab Republic of Egypt up to 1975. Cairo: Ministry of Civil Aviation, Meteorological Authority, General Organization for Governmental Printing Office. 433 pp.

Boulos, L. 1995. Flora of Egypt: CheckList. Al Hadara Publishing, Cairo. $283 \mathrm{pp}$.

1999. Flora of Egypt (Azollaceae-Oxalidaceae). Vol I. Al Hadara Publishing, Cairo. 419 pp.

2000. Flora of Egypt (Geraniaceae-Boraginaceae). Vol II. Al Hadara Publishing, Cairo. 352 pp.

2002. Flora of Egypt (Verbenaceae-Compositae). Vol III. Al Hadara publishing, Cairo. 373 pp.

El-Bakry, A.A.H. 1982. Studies on plant life in the Cairo-Ismailia region. M.Sc. Thesis, Faculty of Science, Cairo University, Cairo, pp. 351.

El-Demerdash, M.A. 1984. Ecological studies on Juncus plants. Ph.D. Thesis, Faculty of Science, Mansoura University, Mansoura.156 pp. , Hosni, H.A. \& Al-Ashri, N. 1997. Distribution of weed communities in the northeast of Nile Delta, Egypt. Feddes Repertorium 108: 219-232.

El-Gabaly, M.M., Gewiafel. I.M., Hassan, M.V. \& Rozanov, B.G. 1969. Soil map and land resources of U.A.R. Institute of Land Reclamation Research Bulletin, Alexandria University 22: 1-14. 
El-Halawany, E.F. 2000. Flora and vegetation of date palm orchards in the Nile Delta, Egypt. Proceedings of the $1^{\text {st }}$ International Conference on Biological Sciences, Faculty of Science, Tanta University, Tanta 1: 266-283.

El-Kholi, A.A. 1989. Biological and ecological studies of Myriophyllum spicatum L. as a basis for a better control. M.Sc. Thesis, Institute of African Studies, University of Cairo, Cairo.

El-Shayeb, F.M. 1984. Studies on the reproductive capacity of major weeds in cultivated land in Menoufia Governorate. M.Sc. Thesis, Faculty of Science, Menoufia University, Menoufia. 397 pp.

1989. Studies on the weed flora of the Nile Delta. Ph.D. Thesis, Faculty of Science, Menoufia University, Menoufia. 429pp.

El-Sheikh, M.A. 1989. A study of the vegetation environmental relationships of the canal banks of the middle Delta region. M.Sc. Thesis, Faculty of Science, Tanta University, Tanta. 139 pp. 1996. Ruderal plant communities of the Nile Delta region. Ph.D., Thesis, Faculty of Science Tanta University, Tanta, pp.173.

Fayed, A.A. 1985. The distribution of Myriophyllum spicatum L. in the inland waters of Egypt. Folia Geobotanica et Phytotaxonomica 20: 197-199.

Goma'a, N.H. 2002. Ecology of vegetation and weed diversity in Beni-Suef Governorate. M.Sc. Thesis, Faculty of Science, Cairo University (Beni-Suef branch), Beni-Suef. 127 pp.

Hill, M.O. 1979a. TWINSPAN-A FORTRAN Program for Arranging Multivariate Data in Order Two-Way Table by Classification of Individuals and Attributes. Cornell University, Ithaca, New York. 52 pp.

1979b. DECORANA-A FORTRAN Program for Detrended Correspondence Analysis and Reciprocal Averaging. Cornell University, Ithaca, New York. 90pp.

Hussein, T.G. 2000. Studies on the River Nile vegetation in El-Kahira ElKobra. M.Sc. Thesis, Faculty of Science, Helwan University, Helwan. 202pp.

Kent, M. \& Coker, P. 1992. Vegetation Description and Analysis: A Practical Approach. John Wiley and Sons, Chichester. 363 pp.

Khedr, A.A. \& El-Demerdash, M.A. 1995. Distribution and environmental relations of the aquatic macrophytes in the northeast section, Nile Delta, Egypt. Proc. $5^{\text {th }}$ International Conference (Environmental 
Protection is a Must). National Institute of Oceanography, Alexandria: 197-223.

\& Zahran, M.A. 1999. Comparative study on the plant life of two Mediterranean Deltaic lakes in Egypt. Assuit University Bulletin of Environmental Research 2 (1): 1-14.

Kruscal, J. B. 1964. Nonmetric multidimensional scaling: A numerical method. Psychometrika 29: 115-129 .

Magurran, A.E. 1988. Ecological Diversity and its Measurements. Princeton University Press. Princeton, New Jersey, pp. 179.

Mohler, C.L. \& Liebman, M. 1987. Weed productivity and composition in sole Crops and intercrops of barely and field pea. Journal of Applied Ecology 24: 685-699.

Murphy, K.J., Rorslett, B. \& Springuel, I.V. 1990. Strategy analysis of submerged lake communities: an international example. Aquatic Botany 36: 303-323.

Pielou, E.C. 1975. Ecological Diversity. A Wiley-Interscience Publication, New York, pp.165.

Radosevich, S.R. \& Holt. J.S. 1984. Weed Ecology. John Wiley and Sons, New York.

Serag, M.S. 1991. Studies on the ecology and control of aquatic and canal bank weeds of the Nile Delta, Egypt. Ph.D. Thesis, Faculty of Science, Mansoura University, Mansoura.

Shaltout, K.H. \& El-Fahar, R.A. 1991. Diversity and phenology of weed communities in the Nile Delta region. Journal of Vegetation Science 2: 385-390.

\& El-Halawany, E.F. 1993. Vegetation analysis of the irrigation and drainage canals in eastern Saudi Arabia. Journal of University of Kuwait (Science) 20: 261-273.

\& El-Sheikh, M.A. 1991. Gradient analysis of canal vegetation in the Nile Delta region. Feddes Repertorium 102: 639645.

\& El-Sheikh, M.A. 1993. Vegetation-environment relations along watercourses in the Nile Delta region. Journal of Vegetation Science 4: 567-570.

\& El-Sheikh, M.A. 2003. Vegetation of the urban habitats in the Nile Delta region, Egypt. Urban Ecosystems 6: 205-221. 
\& Sharaf El-Din, A. 1988. Habitat types and plant communities along A transect in the Nile Delta region. Feddes Repertorium 99: 153-162.

, El-Kady, H.F. \& Al-Sodany, Y.M. 1995. Vegetation analysis of the Mediterranean region of Nile Delta. Vegetatio 116: 7383.

, Sharaf El-Din, A. \& El-Fahar, R.A. 1992. Weed communities of the common crops in the Nile Delta region. Flora 187: 329-339.

Sharaf El-Din, A. \& El-Fahar, R.A. 1994. Species richness and phenology of vegetation along irrigation canals and drains in the Nile Delta, Egypt. Vegetatio 112: 35-43.

Sheded, M.G. \& Turki, Z.A. 2000. Weed communities in south Nile Delta. Proceedings of the $1^{\text {st }}$ International Conference on Biological Sciences, Faculty of Science, Tanta University, Tanta 1: 171-182.

Shehata, M.N. 1994. Ecological studies on Vossia cuspidata (Roxb.) Griff. in the Nile Delta of Egypt. Egyptian Journal of Botany 36 (1): 34-51.

\& El Fahar, R.A. 2000. The vegetation of reclaimed areas in Salhiya region. Proceedings of the $1^{\text {st }}$ International Conference on Biological Sciences, Faculty of Science, Tanta University, Tanta, 1: 315-332.

Spink, A.J. 1992. The ecological strategies of aquatic Ranunculus species.

Ph.D. Thesis, University of Glasgow. Glasgow.

Springuel, I.V. 1981. Studies on the natural vegetation of the islands of the First Cataract at Aswan, Egypt. Ph.D. Thesis, Aswan, Faculty of Science, Assiut University, Assiut. 184pp.

1987. Plant life in Nubia. V. Aquatic plants in Egyptian Nubia. Aswan Science Technology Bulletin 8: 185-221.

\& Murphy, K.J. 1990. Euhydrophytes of Egyptian Nubia. Aquatic Botany 37: 17-25.

SPSS 1999. SPSS Software Package, Version 10. SPSS Incorporation, Chicago.

Täckholm, V. 1974. Students' Flora of Egypt, (2 ${ }^{\text {nd }}$ ed.). Cairo University Press, Cairo. 888pp.

Tadros, T.M. \& Atta, B.A.M. 1958. The plant communities of barley fields and uncultivated desert areas of Mareotis (Egypt). Vegetatio 8: 161175. 
46

K.H. Shaltout et al.

Whittaker, G.H. 1972. Evolution and measurements of species diversity. Taxon 21: 213-251.

Zahran, M.A. \& Willis A.J. 1992. The Vegetation of Egypt. Chapman \& Hall, London. 424 pp.

\& Willis A.J. 2003. Plant Life in the River Nile in Egypt. Mars Publisher House, Riyadh. 531 pp.

Zaki, H. 1947. Centennial Anniversary of El-Qanatir El- Khairiya, 18471947. El-Amiyria Press, Cairo. 40 pp. (in Arabic). 